\title{
Multiple Modes of N-Type Calcium Channel Activity Distinguished by Differences in Gating Kinetics
}

\author{
Anne H. Delcour, ${ }^{a}$ Diane Lipscombe, ${ }^{b}$ and Richard W. Tsien \\ Department of Molecular and Cellular Physiology, Beckman Center, Stanford University Medical Center, Stanford, \\ California 94305
}

In many neurons, $\mathrm{N}$-type $\mathrm{Ca}^{2+}$ channels are a major $\mathrm{Ca}^{2+}$ entry pathway and strongly influence neurotransmitter release. We carried out cell-attached patch recordings (110 $\mathrm{mm} \mathrm{Ba}^{2+}$ as charge carrier) to characterize the rapid opening and closing kinetics of $\mathrm{N}$-type $\mathrm{Ca}^{2+}$ channel gating in frog sympathetic neurons. Single channels display at least three distinct patterns of gating, characterized as low-, medium-, and high- $p_{o}$ modes on the basis of channel open probability $\left(p_{o}\right)$ during depolarizing pulses to $-10 \mathrm{mV}$. Spontaneous transitions from one mode to another are infrequent, with an exponential distribution of dwell times and mean sojourns of $\sim 10 \mathrm{sec}$ in each mode. Thus, a channel typically undergoes hundreds or thousands of open-closed transitions in one mode before switching to a different mode. Transitions between modes during a depolarization were occasionally detected, but were rare, as expected for infrequent modal switching. Within each mode, the activation kinetics were well described by a simple scheme (C2-C1-O), as previously reported for other types of $\mathrm{Ca}^{2+}$ channels. Rate constants are strikingly different from one mode to another, giving each mode its own characteristic kinetic signature. The gating behavior at $-10 \mathrm{mV}$ ranges from brief openings ( $\sim 0.3 \mathrm{msec}$ ) and long closures (10-20 msec) for low- $p_{0}$ gating to long openings ( $3 \mathrm{msec}$ ) and brief closures ( $\sim 1 \mathrm{msec}$ ) for high-

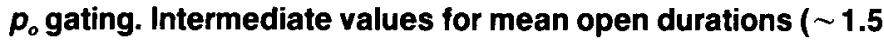
$\mathrm{msec}$ ) and mean closed durations ( $\sim 3 \mathrm{msec}$ ) were found for medium- $p_{o}$ gating. In addition to being kinetically distinct, channel openings in the low- $p_{o}$ mode often exhibit a unitary current $\sim 0.2 \mathrm{pA}$ larger than in the medium- or high- $p_{\mathrm{o}}$ mode. Each mode is characterized by its own voltage dependence: activation occurs at relatively negative potentials and is most steeply voltage dependent in the high- $p_{0}$ mode, while activation requires very strong depolarizations and is weakly voltage dependent in the low- $p_{0}$ mode. The proportion of

\footnotetext{
Received Mar. 26, 1992; revised July 10, 1992; accepted July 15, 1992.

We are grateful to Keith Bley and Pedro Rivas for their participation in early stages of this project, Ji-Fang Zhang for carrying out Monte Carlo simulations, Jian Yang for access to experimental data, and David Friel and Felix Schweizer for valuable assistance and advice on computer programs. We thank Richard Aldrich, Ruth Lagnado, and David Friel for helpful comments on the manuscript. This work was supported by an NRSA award (A.H.D.), a fellowship from the American Heart Association (D.L.), U.S. Public Health Service Grants NS24067 and HLI3156 (R.W.T.), and a National Institutes of Mental Health Silvio Conte Center of Neuroscience Research (MH 48108).

Correspondence should be addressed to Richard $W$. Tsien at the above address. ${ }^{a}$ Present address: Department of Biology, University of Houston, Houston, TX 77204.

'Present address: Section of Physiology, Box GB302, Brown University, Providence, RI 02912.

Copyright (C) 1993 Society for Neuroscience $0270-6474 / 93 / 130181-14 \$ 05.00 / 0$
}

time spent in the individual modes varies greatly from one patch to another, suggesting that modal gating may be subject to cellular control.

[Key words: ion channel, $\mathrm{Ca}^{2+}$ channel, gating modes, activation kinetics, sympathetic neurons, GTP-binding proteins]

$\mathrm{N}$-type $\mathrm{Ca}^{2+}$ channels are a class of voltage-gated $\mathrm{Ca}^{2+}$ channels found almost exclusively in the nervous system. They are of interest for a number of reasons: they are the major pathway for $\mathrm{Ca}^{2+}$ entry in several different types of neuron (Tsien et al., 1988; Bean, 1989a; Hess, 1990), they can play a dominant role in controlling neurotransmitter release (Hirning et al., 1988; Stanley, 1991; Miller, 1992), and they are strongly modulated by neurotransmitters and other ligands (e.g., Tsien et al., 1988; Carbone and Swandulla, 1989; Beech et al., 1992).

Knowledge about $\mathrm{N}$-type channels has grown steadily since their initial description in chick sensory neurons (Nowycky et al., 1985a; Aosaki and Kasai, 1987; Fox et al., 1987). Channels with similar although not identical properties have been found in rat sympathetic neurons and PC12 cells (Hirning et al., 1988; Plummer et al, 1989) and a large variety of central neurons (Regan et al., 1991). Within these preparations, N-type channels exhibit a set of characteristics that distinguish them from other voltage-dependent $\mathrm{Ca}^{2+}$ channels (e.g., T-, L-, and P-type). In general, N-type channels require relatively strong depolarizations for activation, but are prone to inactivate during pulses to weakly depolarized potentials; they are blocked by $\omega$-conotoxin but not by dihydropyridines or $\omega$-agatoxin IVA. Their unitary $\mathrm{Ba}^{2+}$ conductance is generally less than that of L-type channels but greater than T-type channels. A combination of these discriminatory parameters is usually sufficient to allow a reliable distinction between $\mathrm{N}$-type and L-type or P-type channels (see Bean, 1989a; Hess, 1990; Tsien et al., 1991).

Less is known about the gating properties of $\mathrm{N}$-type channels than L-type channels. Whole-cell recordings have provided information about $\mathrm{N}$-type channel activation, inactivation, and pharmacology (e.g., Jones and Marks, 1989a,b). Unitary recordings have revealed variable inactivation rates of $\mathrm{N}$-type channels (Plummer and Hess, 1991). However, no description of rapid activation kinetics is available at the level of single $\mathrm{N}$ type channels.

Studies of rapid gating kinetics are particularly interesting because there have been repeated suggestions that $\mathrm{N}$-type channels show more than one mode of gating. Shifts between "willing" and "reluctant" gating modes have been hypothesized to account for changes in voltage dependence of activation during norepinephrine inhibition (Bean, 1989b) and the relief of this 
A

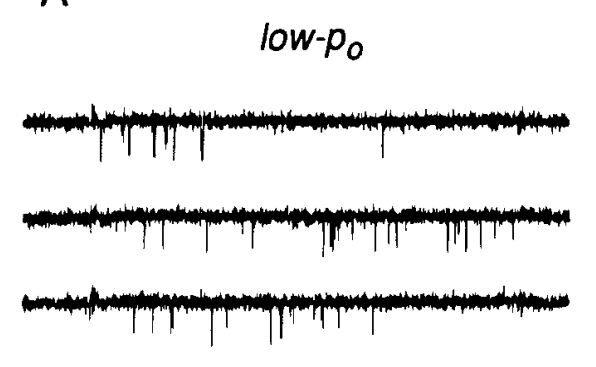

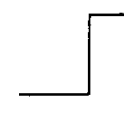

B
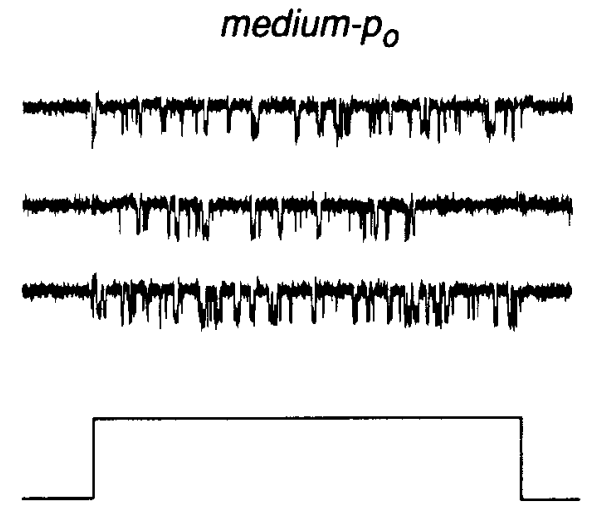

C

high-po

mom

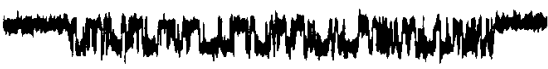

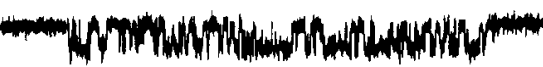

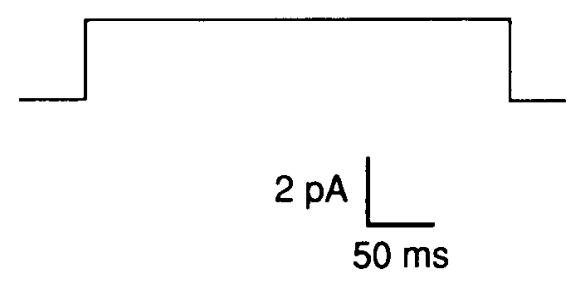

Figure 1. Multiple kinetic patterns of N-type calcium channel gating: leak-subtracted traces selected from a single-experiment to illustrate gating in the low-, medium-, and high- $p_{t}$ modes. The voltage protocol, displayed at the bottom of each panel, was a depolarization from $-80 \mathrm{mV}$ to -10 $\mathrm{mV}$. Experiment dl9c.

inhibition after strong depolarizing prepulses (Elmslie et al., 1990; Kasai, 1992). This modal scheme has also been invoked to explain calcium current facilitation (Ikeda, 1991). These hypotheses for $\mathrm{N}$-type channels find some precedence in previous analysis of modes of gating of L-type channels (Hess et al., 1984; Nowycky et al., 1985b). Modal gating is inferred when a channel changes its kinetic behavior suddenly and maintains it for several seconds in the absence of a change in test potential and bathing solutions. In the case of L-type channels, shifts between gating modes occur during stimulation of $\mathrm{Ca}^{2+}$ currents by dihydropyridines (Hess et al., 1984), $\beta$-adrenergic agonists (Yue et al., 1990), and the activity-dependent $\mathrm{Ca}^{2+}$ channel potentiation (Pietrobon and Hess, 1990). Previous single-channel recordings of $\mathrm{N}$-type channels suggested the presence of two distinct open states (Lipscombe et al., 1989). We have now used high-resolution single-channel analysis to establish whether $\mathrm{N}$-type channels show discrete modes of gating and to determine the kinetic properties within each mode.

This article focuses on analysis of opening and closing kinetics of N-type channels in frog sympathetic neurons. This a particularly favorable system since the cells lack T-type channels, and $\mathrm{N}$-type channels greatly outnumber L-type channels. We found that a single $\mathrm{N}$-type channel can undergo spontaneous shifts between three gating modes, each with markedly different mean open and closed times at a fixed test potential and different voltage dependence. Typically, the channel remains in the same kinetic pattern for periods ranging from several seconds to minutes, a time scale scveral orders of magnitude slower than required for individual open-closed transitions. Interestingly, the unitary flux through the channel can be significantly larger when the channel is in the mode with the lowest open probability $\left(p_{o}\right)$. The differences in kinetics and unitary conductance support the idea that modes reflect fundamentally different configurations of the channel.

\section{Materials and Methods}

Cell culture. Sympathetic neurons were dissociated from the entire sympathetic chains of adult bullfrogs (Rana catesbeiana) following the procedure of Lipscombe et al. (1988) and maintained in culture at $16^{\circ} \mathrm{C}$ or $23^{\circ} \mathrm{C}$ for up to $15 \mathrm{~d}$. The cells were plated in noncoated plastic dishes
(Corning) and supplemented with growth medium [73\% L-15 medium (GIBCO), 7\% Nu-Serum (Collaborative), $10 \mathrm{~mm}$ glucose, $1 \mathrm{~mm} \mathrm{CaCl}$, $100 \mathrm{U} / \mathrm{ml}$ penicillin, $100 \mathrm{mg} / \mathrm{ml}$ streptomycin]. After a few days, healthy cells developed processes that made contacts with each other.

Patch-clamp recordings. Single-channel measurements were made according to standard techniques (Hamill et al., 1981). The membrane potential was zeroed with the following bath solution: $140 \mathrm{mM} \mathrm{K}$-aspartate, $10 \mathrm{~mm}$ EGTA, $10 \mathrm{~mm}$ glucose, $1 \mathrm{~mm} \mathrm{MgCl}_{2}, 10 \mathrm{~mm}$ HEPES ( $\mathrm{pH}$ adjusted to 7.3 with $\mathrm{KOH}$ ). Pipcttes containcd solutions of 110 $\mathrm{mM} \mathrm{BaCl}, 10 \mathrm{~mm}$ tetraethylammonium chloride (TEA-Cl), $5 \mathrm{~mm} \mathrm{4-}$ aminopyridine, and $5 \mathrm{~mm}$ HEPES ( $\mathrm{pH}$ adjusted to 7.3 with TEA-OH), and had resistances of 7-15 M . Current was recorded in the cellattached configuration with an Axopatch-1C (Axon Instruments) and filtered at $2 \mathrm{kHz}$ (8-pole Bessel filter, Frequency Devices). For most of the experiments, the membrane potential of the patch was held at -80 $\mathrm{mV}$, but occasionally it was changed to $-40 \mathrm{mV}$ to identify $\mathrm{N}$-type $\mathrm{Ca}$ channels through their voltage-induced inactivation. Command pulses of $312 \mathrm{msec}$ in duration were delivered every $4 \mathrm{sec}$, and current was sampled every $100 \mu \mathrm{sec}$ and stored on computer.

Data analysis. Data acquisition, analysis, and simulation were performed with 486-based microcomputers using AXOBAsIC software. Leak and capacitative currents were subtracted from the traces using blank sweeps that were recorded at the same test potential and either averaged or fit by single exponential decays. Channel openings were detected with the half-amplitude criterion. For each sweep, $\left\langle t_{v}\right\rangle$ was calculated as the arithmetic average of all open times, and $\left\langle t_{c}\right\rangle$ as the average of all closed times excluding the first and last closures during the depolarization. The open probability, $p_{o}$, was calculated as $\left\langle t_{o}\right\rangle /\left(\left\langle t_{o}\right\rangle+\left\langle t_{c}\right\rangle\right)$. This is equivalent to the ratio of the total open time during the sweep to the time between the first opening and the completion of the last complete open-closed duty cycle, in which the last closure is assigned a value of $\left\langle t_{r}\right\rangle$. This procedure removes the influence of early inactivation on the estimation of $p_{o}$. Fits of amplitude histograms, open and closed time distributions, and voltage dependence of kinetic parameters were performed with the maximum likelihood algorithm using a Simplex routine (Kowalik and Osborn, 1968; Colquhoun and Sigworth, 1983).

In some experiments, we observed channcl openings of much smaller size than those of typical $\mathrm{N}$-type $\mathrm{Ca}^{2+}$ channels. These events might reflect a subconductance similar to that described for rat sympathetic neurons (Plummer et al., 1989). Such activity could always be clearly distinguished from typical $\mathrm{N}$-type $\mathrm{Ca}$ channel behavior by virtue of its smaller slope conductance $(8 \mathrm{pS})$ and the relatively negative position of its voltage-dependent activation curve. Sweeps that displayed this activity alone or in conjunction with typical $N$-type channel openings were excluded from the analysis presented here. In a few patches, this smaller conductance was found alone for the entirety of the experiment. It is unclear whether the subconductance represents another channel subtype or an infrequently occurring gating mode. 


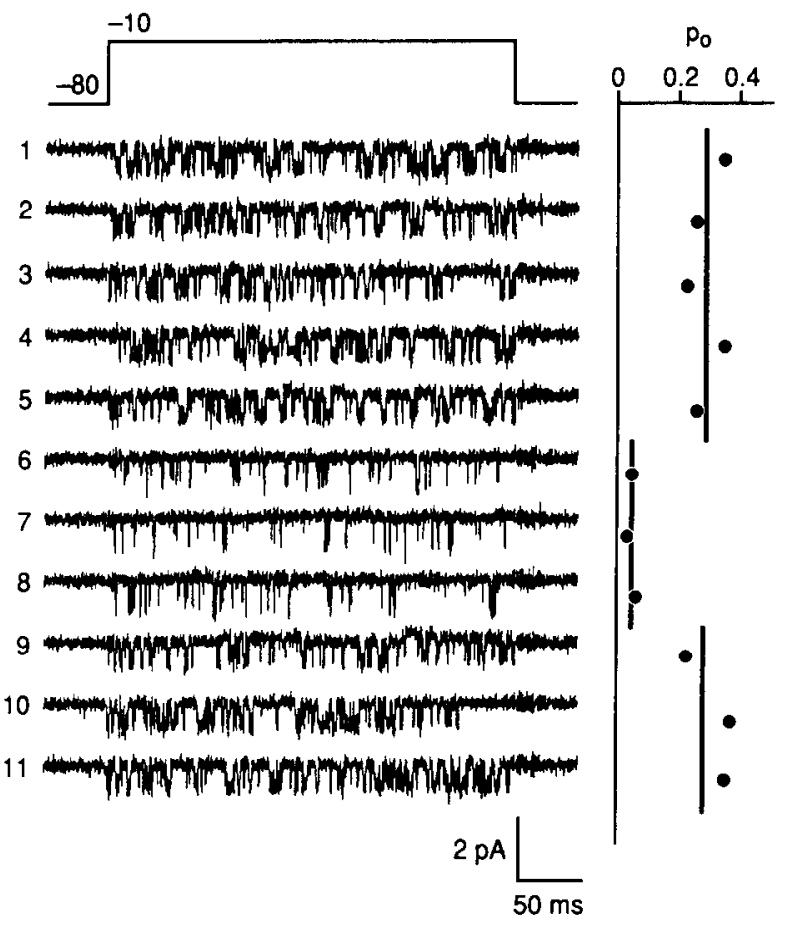

\section{Results}

\section{Different kinetic patterns}

$\mathrm{N}$-type $\mathrm{Ca}^{2+}$ channels display diverse patterns of opening and closing in response to repeated depolarizations to a fixed test potential. As an overview, Figure 1 illustrates recordings of unitary $\mathrm{N}$-type openings that were evoked by $312 \mathrm{msec}$ depolarizing pulses from -80 to $-10 \mathrm{mV}$. Figure $1 A-C$ shows sets of current records, selected to illustrate the different types of gating behavior, where only one channel appears to be active in any swecp. In Figure $1 A$, the kinctic pattern is characterized by brief openings separated by long closures and a low $p_{\sigma}$. In Figure $1 C$, the openings are much longer and the closures much briefer, and consequently, $p_{o}$ is relatively high. Figure $1 B$ shows

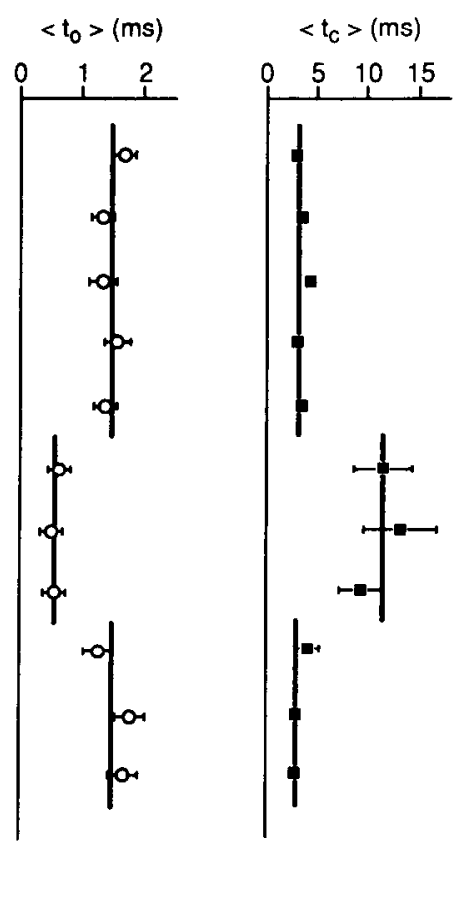

Figure 2. A single N-type channel alternates between the low-and medium$p$, modes. A run of consecutive leaksubtracted sweeps is displayed (left) together with corresponding sweep-bysweep plots of open probability $p_{\text {(, }}$, mean open lime $\left\langle t_{s}\right\rangle$, and mean closed time $\left\langle i_{c}\right\rangle$ (arithmetic averages, \pm SEM). $p_{0}$ is calculated as $\left\langle t_{o}\right\rangle /\left(\left\langle t_{o}\right\rangle+\left\langle t_{t}\right\rangle\right)$ as described in Materials and Methods. Voltage protocol is shown as the top trace. Experiment d19d.

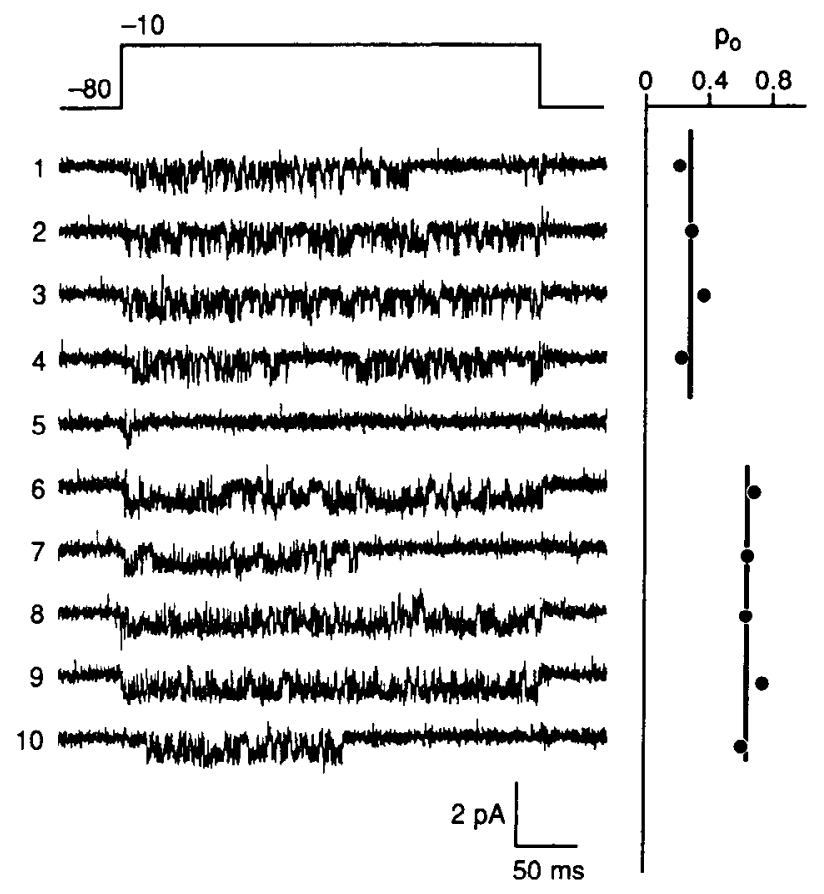

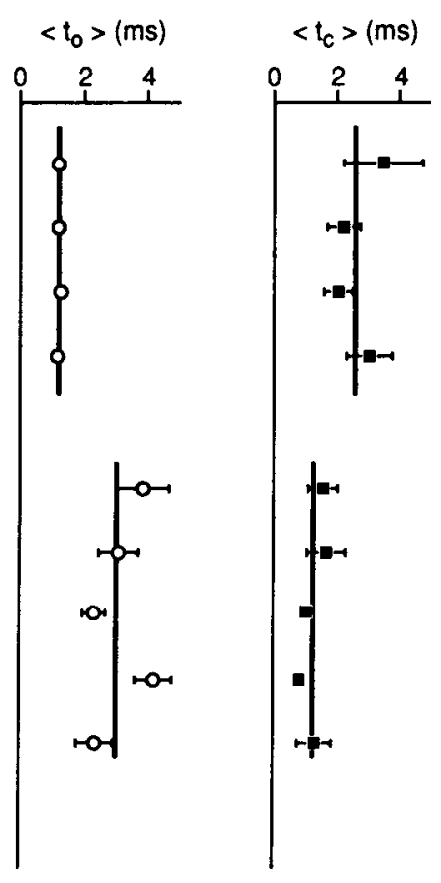

Figure 3. A single N-type channel shifts from the medium- to the high- $p_{o}$ gating mode. Sweep-by-sweep plots of $p_{i,},\left\langle t_{v}\right\rangle$, and $\left\langle t_{c}\right\rangle$ are displayed adjacent to the corresponding sweep. $p_{0}$ is calculated as in Figure 2. Experiment ada 18. 

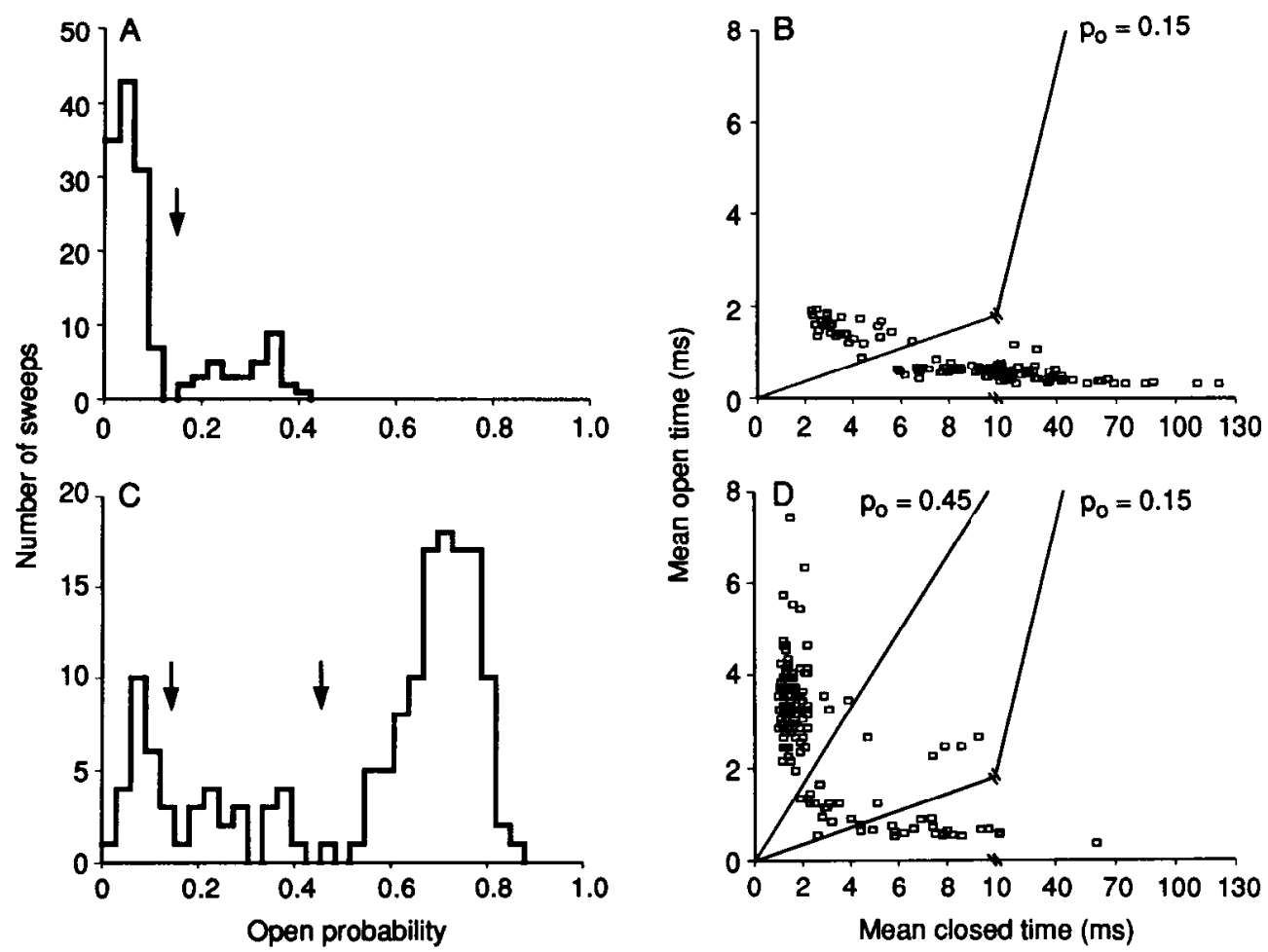

Figure 4. Overall kinetic behavior in two exemplar experiments. $A$ and $B$ show kinetic parameters determined for the experiment illustrated in Figure 2; $C$ and $D$, for the experiment in Figure 3. $A$ and $C$, Histograms of sweep $p_{o}$. The ordinate plots the number of sweeps per bin (bin width $=0.03$ ). The arrows mark $p_{o}$ values of 0.15 and 0.45 , chosen as cutoffs to separate peaks of low-, medium-, and high- $p_{w}$ behavior. $B$ and $D$, Plots of $\left\langle t_{o}\right\rangle$ against $\left\langle t_{c}\right\rangle$. Each symbol represents $\left\langle t_{o}\right\rangle$ and $\left\langle t_{c}\right\rangle$ values determined for an individual sweep. Note that the abscissa plots time on a contracted scale beyond the break at $10 \mathrm{msec}$. In $B$, the broken diagonal line corresponds to $p_{o}=0.15$. Note that this separates the sweeps into two distinct populations. $D$ includes an additional diagonal line corresponding to $p_{o}=0.45$, which lies below an additional cluster of data points corresponding to high- $p_{o}$ gating. Between $p_{o}=0.45$ and $p_{o}=0.15$ are four data points representing sweeps with $\left\langle t_{o}\right\rangle \sim 2 \mathrm{msec}$ and $\left\langle t_{c}\right\rangle$ ranging from 6 to $10 \mathrm{msec}$. Although these sweeps fell in the category of "medium- $p_{o}$," they each showed a transition between the low- and high- $p_{o}$ modes.

closed time $\left\langle t_{c}\right\rangle$. Distinctions between gating patterns of low $p_{o}$ and medium $p_{o}$ are illustrated in Figure 2. The pattern of gating appears relatively uniform up to the fifth sweep, with $p_{o} \sim 0.3$. In the next threc swecps, $p_{o}$ falls approximately sixfold to $\sim 0.05$, but recovers abruptly to $\sim 0.3$ during the ninth sweep. The changes in channel $p_{o}$ are the result of marked changes in both open and closed durations: more than a twofold difference in $\left\langle t_{c}\right\rangle$ and a nearly fourfold difference in $\left\langle t_{o}\right\rangle$. These records are representative of the gating behavior seen throughout the entire recording $(\sim 35 \mathrm{~min})$. The vast majority of the changes in gating pattern took place during the interval between depolarizations, rather than during the pulses themselves (see Slow transitions between gating modes, below).

The distinction between gating patterns of medium- $p_{o}$ and high- $p_{o}$ activity is illustrated in Figure 3 . These consecutive sweeps originate from another single $\mathrm{N}$-type $\mathrm{Ca}^{2+}$ channel patch. Clear differences between the kinetic pattern of the first four sweeps and the last five sweeps are highlighted by the accompanying plots of $p_{o},\left\langle t_{o}\right\rangle$, and $\left\langle t_{c}\right\rangle$. The first four sweeps show $p_{o}$ values close to 0.3 , similar to the values found for groups of sweeps in Figure 2. After a nearly blank record, the $p_{o}$ shifts upward to a mean $p_{o}$ of 0.6 during the sixth sweep. This change in $p_{o}$ is paralleled by a doubling of $\left\langle t_{o}\right\rangle$ and a twofold decrease in $\left\langle t_{c}\right\rangle$.

Each of the data points for $\left\langle t_{o}\right\rangle$ and $\left\langle t_{c}\right\rangle$ in Figures 2 and 3 is associated with an SEM (in some cases the error bars are so small that they lie within the symbols). The apparent differences in mean open or closed durations between modes were found to be highly significant. For example, consider the estimates of $\left\langle t_{o}\right\rangle$ for medium- and high- $p_{o}$ activity in Figure 3. The highest estimate of $\left\langle t_{o}\right\rangle$ in the medium- $p_{o}$ records (sweep 3) was $1.32 \pm$ $0.19 \mathrm{msec}(N=92)$, and the lowest value for $\left\langle t_{o}\right\rangle$ in the high$p_{o}$ traces (sweep 8 ) was $2.08 \pm 0.28 \mathrm{msec}(N=98)$. The null hypothesis that both samples are drawn from the same parent distribution can be rejected at the $p<0.05$ level (Student's $t$ test). Estimates of $\left\langle t_{c}\right\rangle$ for the same sweeps $(2.05 \pm 0.31$ and $1.05 \pm 0.15)$ are different at the $p<0.01$ level. Likewise, a comparison of $\left\langle t_{c}\right\rangle$ for low- and medium- $p_{o}$ sweeps (e.g., sweeps 6 and 3 in Fig. 2) yielded qualitatively similar conclusions. While the number of open and closed events was smaller for low- $p_{o}$ sweeps $(N \sim 35)$, differences in $\left\langle t_{o}\right\rangle$ and $\left\langle t_{c}\right\rangle$ between these modes were nevertheless significant $(p<0.05, p<0.02$, respectively). The overall conclusion is that medium- $p_{o}$ gating may be readily distinguished from either low- $p_{o}$ or high- $p_{o}$ activity by consideration of either $\left\langle t_{o}\right\rangle$ or $\left\langle t_{c}\right\rangle$.

This study was mainly directed at studying rapid transitions between closed and open states. However, it should be noted that variations in the rate of inactivation may also be seen. For example, in some of the traces in Figure 3, channel openings disappear well before the end of the depolarizing pulse. Evidently, single N-type channels in frog sympathetic neurons can spontaneously switch between relatively rapid inactivation and little or no inactivation (see Kongsamut et al., 1989), as previously reported in rat sympathetic neurons (Plummer and Hess, 1991). The variations in inactivation rate appear independent of modal changes in activation kinctics (sce also Rittenhousc ct al., 1991). For the purposes of studying rapid opening and closing kinetics, we minimized the influence of variable inactivation 
kinetics by excluding from the kinetic analysis the last closed interval during a depolarizing pulse.

\section{Classification of sweeps into groups}

Data from the same single-channel patches were used to construct histograms showing distributions of sweep $p_{o}$ (Fig. 4A,C). The distribution obtained from the experiment of Figure 2 shows two distinct peaks (Fig. $4 A$ ), corresponding to the two kinds of sweeps that were separated by visual inspection. This type of distribution was seen in less than $20 \%$ of the cells. Figure $4 C$ shows the distribution of $p_{0}$, for all the sweeps in the experiment of Figure 3. This histogram is dominated by a large peak centered around $p_{0}=0.7$ that includes most of the sweeps in this experiment. The population of sweeps in the high- $p_{o}$ mode stands apart from the rest of the distribution. The remainder of the sweeps appear in a well-defined peak centered around $p_{o}=0.08$ (corresponding to the low- $p_{\text {o }}$ peak in Fig. $4 A$ ), and a rather broad distribution of sweeps of intermediate $p_{v}$. $p_{o}$ cutoffs of 0.15 and 0.45 (Fig. $4 A, C$, arrows) are chosen to separate sweeps of low-, medium-, and high- $p_{0}$ behavior.

Another way of representing the nonhomogeneous gating behavior of the channel is to plot $\left\langle t_{0}\right\rangle$ against $\left\langle t_{c}\right\rangle$ on a sweep-bysweep basis (Fig. $4 B$ ). In this case, a probability cutoff of 0.15 is represented by a diagonal through the origin. The data points below the diagonal (short $\left\langle t_{t}\right\rangle$, long $\left\langle t_{c}\right\rangle$ ) correspond to the group of sweeps with $p_{0}<0.15$; the points above the diagonal represent sweeps with $p_{0}>0.15$.

If one considers all the sweeps for $\left\langle t_{i}\right\rangle$ up to $10 \mathrm{msec}$, there is a clear negative correlation between $\left\langle t_{o}\right\rangle$ and $\left\langle t_{c}\right\rangle$ : short openings appear to be strongly associated with long closures. This negative correlation is not consistent with a simple unbranched kinetic scheme. It can be accommodated by Markovian kinetics, but only if the scheme has a branched topology, with at least one closed state and one open state in each branch (Jackson et al., 1983; McManus and Magleby, 1989). However, if one considers separately the groups on either side of the $p_{0}=0.15$ linc, there is little or no correlation between $\left\langle t_{0}\right\rangle$ and $\left\langle t_{i}\right\rangle$ within each group. The kinetics within each group would therefore be consistent with a simple linear scheme comprised of a series of closed states leading up to a series of one or more open states (C . . C-O-O . . .). For this kind of scheme, the closed times would be distributed independently of the length of the previous openings.

The populations of sweeps defined in Figure $4 C$ were also examined in a plot of $\left\langle t_{o}\right\rangle$ versus $\left\langle t_{c}\right\rangle$ (Fig. $4 D$ ). Here, as in Figure $4 B$, the low- $p_{c}$, sweeps are represented by a horizontal streak of data points below the $p_{o}=0.15$ diagonal and the medium- $p_{0}$ sweeps cluster together above this diagonal. The high- $p_{\text {o }}$ peak is represented as an additional group of points, lying above the $p_{0}=0.45$ diagonal, with $\left\langle t_{c}\right\rangle$ values consistently smaller than 2 msec and $\left\langle t_{a}\right\rangle$ values extending between 2 and $8 \mathrm{msec}$. This kind of behavior was completely absent in Figure $4 B$. Within the population of high- $p_{0}$, points, there is no clear correlation between $\left\langle t_{v}\right\rangle$ and $\left\langle t_{t}\right\rangle$, suggesting once again that the kinetics within the mode can be accounted for by an unbranched series of states (see Simple and consistent gating kinetics within each mode, below). If medium- $p_{o}$ and high- $p_{o}$ sweeps were not separated, the scheme would have to be complicated enough to account for a clear negative correlation between $\left\langle t_{0}\right\rangle$ and $\left\langle t_{c}\right\rangle$.

\section{Slow transitions between gating modes}

As a general rule, channels displayed a mode of gating for several consecutive sweeps before changing spontaneously to another

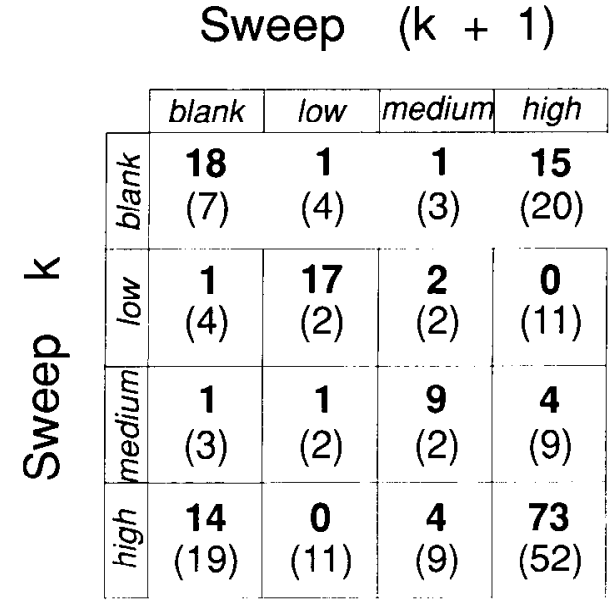

Figure 5. N-type channels tend to display the same pattern of gating from one sweep to the next: contingency table of calcium channel gating from a single-channel patch (ada 18). The gating pattern of sweep ( $k+$ 1 ) is shown in relation to gating of sweep $k$. The observed numbers of sweeps in each category are shown in boldface. The numbers expected for random occurrence are shown below in parentheses. These were calculated as the product of individual modal probabilities times the total number of sweeps.

mode or becoming temporarily inactive. This clustering behavior was analyzed in several ways. One approach, illustrated for a representative experiment in Figure 5, tabulates the number of times that a sweep of a particular type was followed by a sweep of the same type or a different type. A total of 161 sweeps were classified in this way. The boldface number in each box represents the observed number of sweeps of each category, whereas the number in parentheses represents the expected number if each type of gating occurred randomly, independent of the previous history of gating. Examination of the entries on diagonal shows that the observed frequencies of repeats of the same kind of gating are considerably higher than expected if the pattern of gating varied randomly from one depolarization to the next. Analysis with $\chi^{2}$ indicates that the observed frequencies are statistically different from the expected ones at a significance level of $P<0.005$. A highly diagonalized contingency table was obtained in all four experiments for which this type of analysis was performed.

A somewhat different but complementary approach is to subdivide a recording session into runs of sweeps showing the same pattern of gating. Analysis of the lengths of the runs provides direct information about how long a channel remains in a mode of gating before switching to another mode. We distinguished between runs of blank sweeps and of low-, medium-, and high$p_{o}$ modes. For the experiment documented in Figure 5 , the mean numbers of records per run were 1.88 (blanks), 3.00 (low $p_{n}$ ), 1.43 (medium $p_{o}$ ), and 3.5 (high $p_{o}$ ). Given the 4 sec cycle time between sweeps, the mean sojourn in the individual gating modes was of the order of 8-15 sec.

Collected results from a total of four experiments were pooled to yield distributions of run lengths for each of the active modes and for sweeps with no detectable openings (Fig. 6). Each of the histograms is reasonably well fit by a single exponential curve, as expected if the exit rate from an individual mode obeys firstorder kinetics as in a Markov model.

\section{Simple and consistent gating kinetics within each mode}

Separation of the sweeps into separate populations facilitates the analysis of gating kinetics. Figure 7 shows distributions of 
Figure 6. Distributions of run lengths for various modes. A run was defined as a series of consecutive sweeps displaying channel activity in the same mode. $A-C$ show high-, medium-, and low- $p_{o}$ modes, respectively. $D$ displays run lengths for consecutive series of sweeps showing no detectable channel activity (blanks). The histograms were constructed by pooling data from four experiments. The duration of sojourns in various modes can be obtained by multiplying the number of sweeps by 4 sec, the cycle time of the depolarizations. The smooth curves show single exponential fits to the distributions, corresponding to time constants of 6.8 sec (high $p_{o}$ ), $8.4 \mathrm{sec}$ (medium $p_{o}$ ), 5.2 sec (low $p_{o}$ ), and $3.5 \mathrm{sec}$ (blanks).
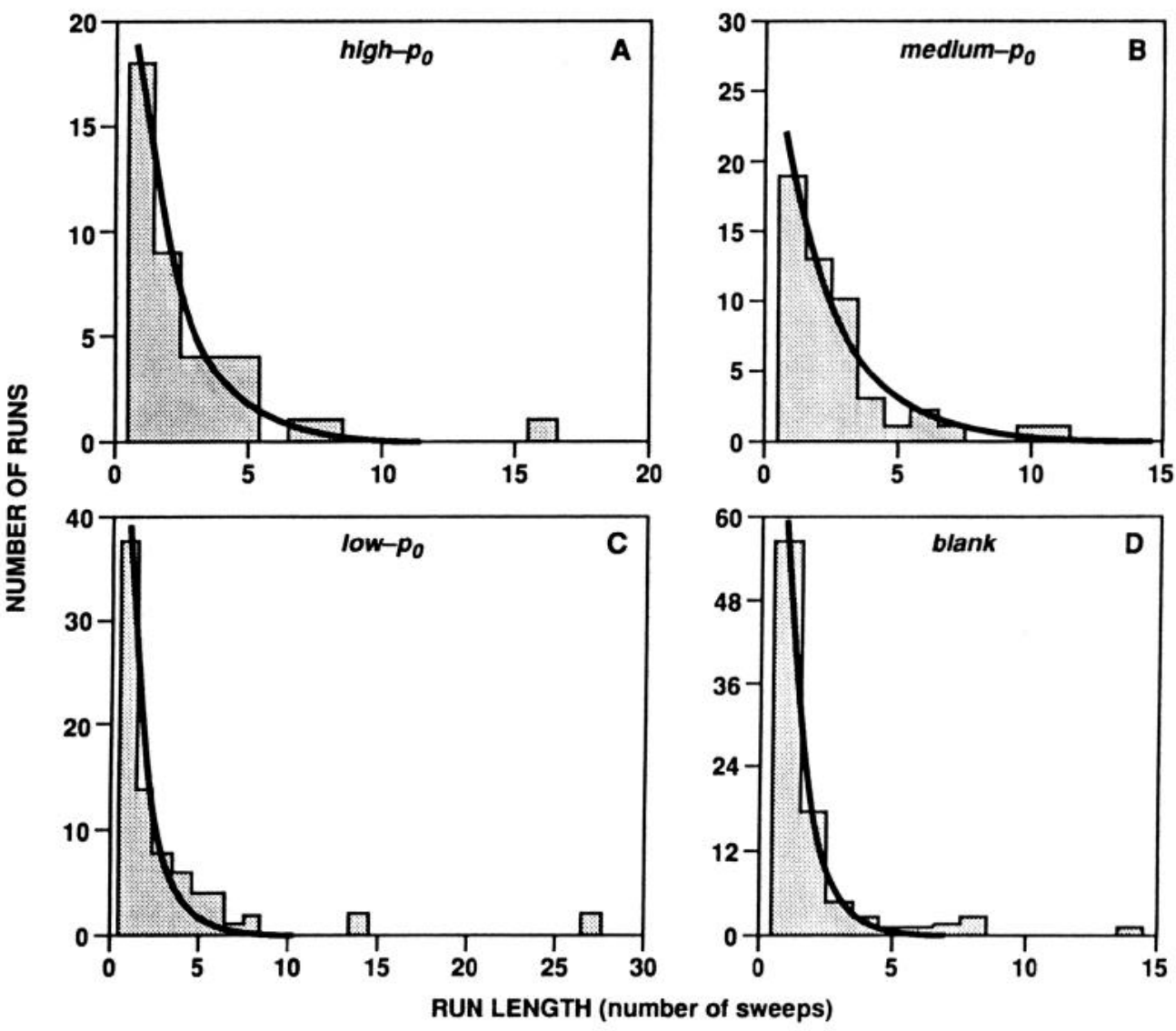

channel open times at $-10 \mathrm{mV}$ for the two exemplar singlechannel experiments (Fig. 4A,C). The leftmost panels show the open time histograms of all sweeps in each of the experiments. In both cases, the distribution is poorly fit by a single exponential, indicating the existence of more than one open state. However, single exponentials proved to be sufficient once the sweeps were broken down into groups according to the mode of gating. In Figure $7 A$, this was accomplished by subdividing the records into low- and medium- $p_{o}$ groups. In Figure $7 B$, subdivision into three modes was necessary; merely removing high- $p_{o}$ sweeps (the most clearly defined group in Fig. 4C) left a distribution of openings that could not be fit by a single exponential (analysis not shown). Further separation between medium- and low- $p_{o}$ sweeps gave three distributions, each conforming to a single exponential. The time constants of the exponentials ranged from $\sim 0.35 \mathrm{msec}$ for the low- $p_{o}$ mode to $3 \mathrm{msec}$ for the high- $p_{o}$ mode.
Figure 7. Separation of modal behavior simplifies analysis of open-closed kinetics: distributions of open times for all openings (left) and for individual modes (labeled low, medium, and high). $A$, Analysis of an experiment in which only low- and medium- $p_{o}$ activity was observed (Fig. 4A,B; experiment dl9d). $B$, Analysis of experiment where all three modes were observed (Fig. $4 C, D$; experiment ada18). The leftmost histograms, made from all openings without modal classification, are poorly fitted by single exponentials. In contrast, histograms constructed from sweeps within the categories of low-, medium-, or high- $p_{o}$ mode are fit very closely by single exponentials. Note that there is close agreement between the experiments in estimated values of exponential time constants. For $A$ and $B$, respectively, $\tau_{\text {low }}=0.32$ and $0.40 \mathrm{msec} ; \tau_{\text {medium }}=1.3$ and $1.2 \mathrm{msec}$. In $B, \tau_{\text {high }}$ is $3.1 \mathrm{msec}$. The relative proportion of detectable openings in each group were $67 \%$ (low) and $33 \%$ (medium) in $A$ and $20 \%$ (low), $8 \%$ (medium), and $72 \%$ (high) in $B$.
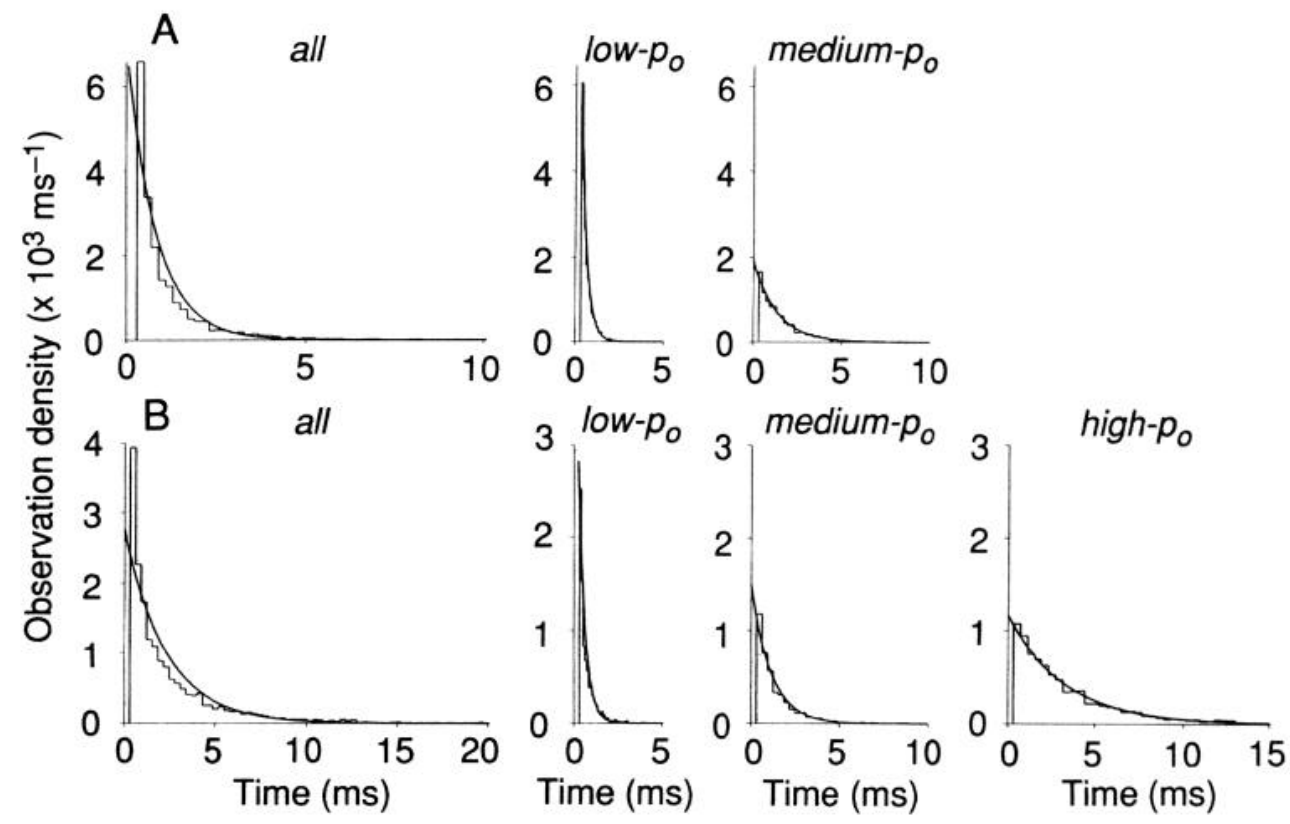

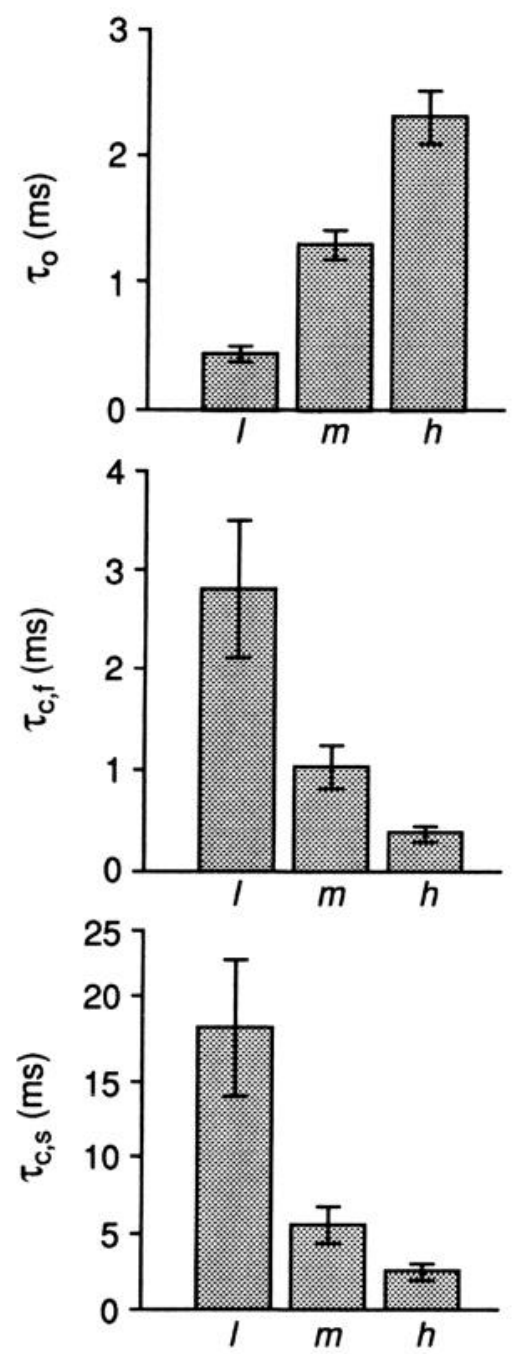

Figure 8. Mean values of open and closed time constants from 13 experiments. Data were collected from exponential fits of the open and closed time distributions after separating the sweeps into the low- $(l)$, medium- $(m)$, and high- $p_{o}(h)$ modes on the basis of cutoffs at $p_{o}=0.15$ and $p_{o}=0.45 . N=7$ for low- $p_{o}$, and $N=11$ for medium and high- $p_{o}$ (the number of entries varies because some experiments displayed too few openings in a particular mode to allow a reliable fit of the distribution).

The relative areas under the individual distributions represent the relative proportion of openings within each mode (see Fig. 7 caption). These exemplar experiments illustrate the general finding that the proportion of time spent in each mode may vary widely from patch to patch. However, in all of our experiments, single exponentials provided good fits to the open time distributions within a mode.

A similar analysis of closed times was carried out after segregation of the sweeps into the three modes. For a given mode, fits to the distributions of closed times required at least two decaying exponentials. This kinetic behavior implies a minimum of two closed states. Thus, a minimal kinetic scheme within each mode can be stated as $\mathrm{C} 2-\mathrm{Cl}-\mathrm{O}$, as found previously for activation kinetics of other types of voltage-gated $\mathrm{Ca}^{2+}$ channels (e.g., Fenwick et al., 1982; Reuter et al., 1982; Brum et al., 1984). Other schemes with more states but the same number of independent rate constants would work equally well (Shuba et al., 1991).

Figure 8 plots collected values of the exponential time con-

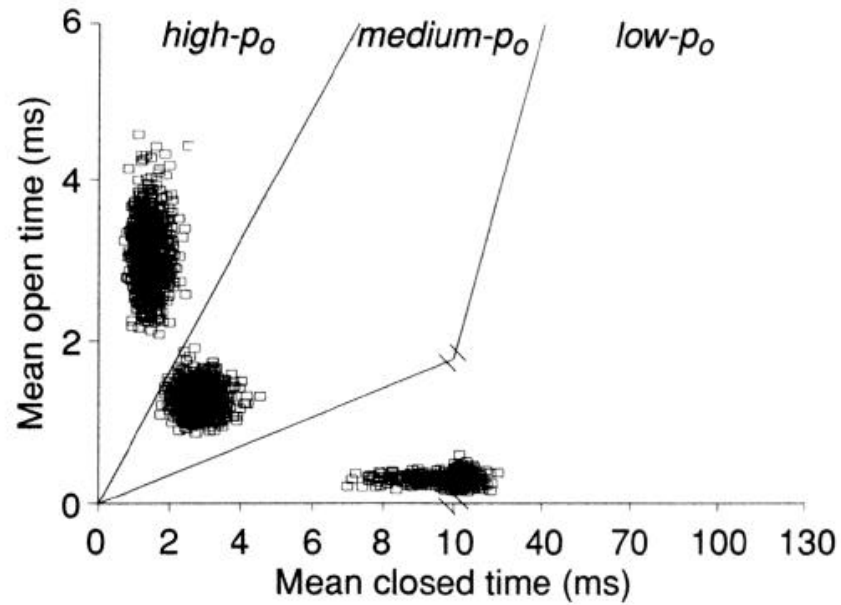

Figure 9. Monte Carlo simulation of kinetic behavior in individual modes: plot of $\left\langle t_{o}\right\rangle$ versus $\left\langle t_{c}\right\rangle$, for simulated sweeps, with 1000 simulated sweeps for each mode. Simulation was based on the kinetic scheme

$$
\mathrm{C} 2 \underset{k_{-1}}{\stackrel{k_{1}}{\rightleftharpoons}} \mathrm{C} 1 \underset{k_{-2}}{\stackrel{k_{2}}{\rightleftharpoons}} \mathrm{O}
$$

with rate constants (in units of $\sec ^{-1}$ ) for individual modes derived from experimental observations. High- $p_{o}$ kinetics were obtained from analysis of the experiment in Figure 3. Kinetics of medium- and low- $p_{o}$ modes were derived from the experiment of Figure 2. Values of $k_{1}, k_{-1}, k_{2}$, and $k_{-2}$ were (in sec ${ }^{-1}$ ), for high- $p_{o}, 667,1311,2137,324$; for medium- $p_{o}$ $1378,1033,633,769$; and for low- $p_{o}, 1163,232,104,3125$.

stants for individual modes (13 experiments). The number of data points for each mode varies because some patches lacked enough sweeps within a particular mode to allow a reliable estimate of the open and closed time constants. The kinetic parameters for the high- $p_{o}$ mode showed relatively little variation from one patch to the next, with an open time constant of about $2.4 \mathrm{msec}$ and fast and slow closed time constants $\left(\tau_{\mathrm{cf}}\right.$, $\tau_{\mathrm{cs}}$ ) of 0.4 and $2.5 \mathrm{msec}$. The medium- $p_{o}$ mode displayed mean values of $\tau_{o}=1.3 \mathrm{msec}, \tau_{\mathrm{cf}}=1.0 \mathrm{msec}$, and $\tau_{\mathrm{cs}}=6 \mathrm{msec}$. Data for the low- $p_{o}$ mode showed the greatest variability from patch to patch; mean values were $\tau_{o}=0.4, \tau_{\mathrm{cf}}=2.8$, and $\tau_{\mathrm{cs}}=18 \mathrm{msec}$.

\section{Monte Carlo simulation of modal analysis}

As a check on whether this analysis can account for the general features of the kinetic behavior, Monte Carlo simulations of channel gating during depolarizing pulses were performed and analyzed by the same procedures used for real data (Fig. 9). The simulations used rate constants derived from analysis of the exemplar experiments, to allow a detailed comparison with Figure $4, B$ and $D$. These rate constants were derived according to standard equations (Fenwick et al., 1982) and are given in the Figure 9 caption. A thousand simulated sweeps were generated for each gating mode. When $\left\langle t_{o}\right\rangle$ is plotted against $\left\langle t_{c}\right\rangle$ for individual sweeps, the data points cluster in well-defined regions, separated by the $p_{o}$ cutoffs of 0.15 and 0.45 . The simulated points reproduce the overall shapes of the experimental clusters, and confirm that they can be reconstructed from three independent gating pathways, each characterized by its own set of rate constants.

The experimental data points are somewhat more scattered than the simulations. This may arise in a number of ways. If the N-type channel undergoes inactivation early during the depolarization, there will be a relatively small number of open and closed time intervals, and therefore a larger variation in $\left\langle t_{o}\right\rangle$ 
A

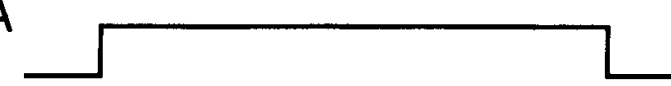

$m$

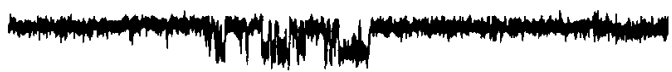

\section{$m$}
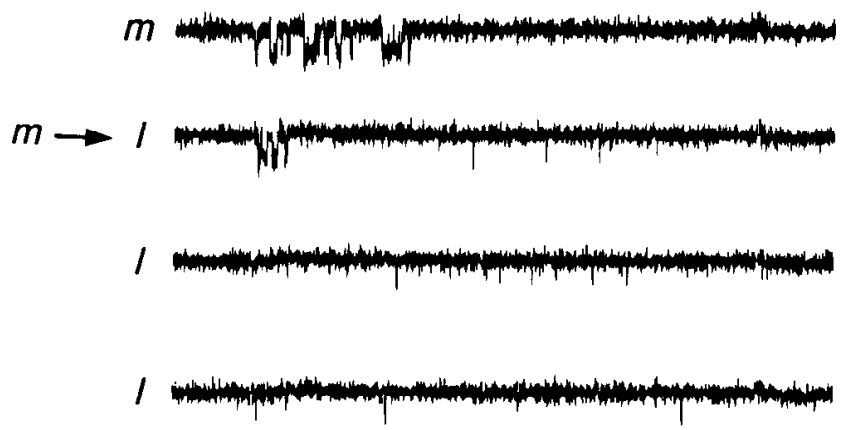

B

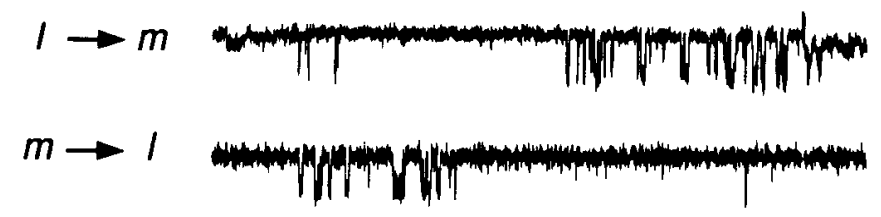

$I \rightarrow n$

$h \rightarrow 1$

mandm

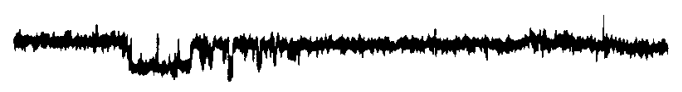

$m \rightarrow h$

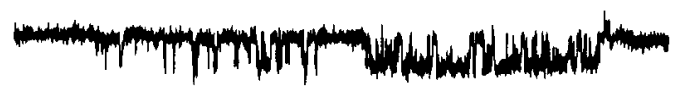

$h \rightarrow m$
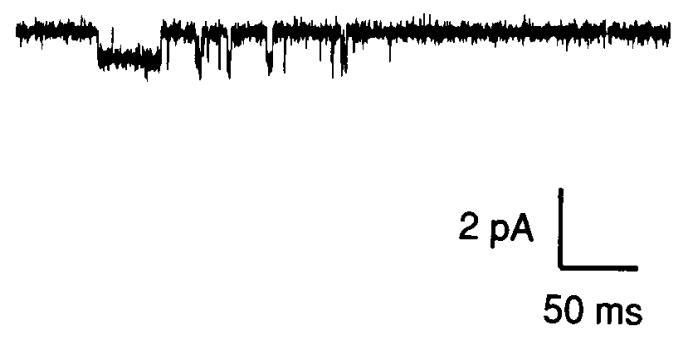

Figure 10. A, Consecutive traces showing a single modal transition (during sweep 4). The gating pattern was characteristic of the medium- $p_{o}$ mode $(m)$ during the preceding 11 sweeps (including sweeps $1-3$ ), and remained as low $p_{o}(l)$ for 9 sweeps after sweep 4 (including $s w e e p s 5$ and 6 ). $B$, Examples of apparent mid-sweep transitions between gating modes. The labels denote our classification of the modal transition. Leak-subtracted sweeps were selected from four experiments. The voltage protocol is shown at the top of $A$ and $B$. The test potential was - $10 \mathrm{mV}$ in all cases, and the holding potential was either $-80 \mathrm{mV}$ or $-70 \mathrm{mV}$.

and $\langle t\rangle$ in the experiment than in the simulation, which ignores inactivation. Likewise, transitions into and out of the inactivated state may occur during experimental depolarizations, but are not modeled in the simulation. Channels may exhibit additional modes, beyond the three considered here (see Discussion). Yet another possibility is that modal transitions may occur partway through the depolarizing pulse.

\section{Modal transitions within individual sweeps}

Some examples of mid-sweep modal transitions are presented in Figure 10, where $A$ shows a sequence of consecutive records of N-type channel gating. During the first three sweeps, the gating pattern is typical of the medium- $p_{o}$ mode (with rather variable inactivation); the gating changes abruptly to the low$p_{0}$, mode partway along sweep 4 and remains so thereafter. Figure $10 B$ provides examples of intermodal transitions encompassing all possible permutations of modes, including low $\rightarrow$ medium, low $\rightarrow$ high, and so on. These records were classified by measuring $p_{o}$ and $\left\langle t_{o}\right\rangle$ over time intervals of homogeneous gating as judged by visual inspection. There was no obvious indication of a graded change in gating properties; the transition seemed to be abrupt.

It was not unusual to find several such transitions in experimental recordings containing hundreds of sweeps. This is roughly as expected if the rate of switching between modes did not depend very strongly on membrane potential over the range between $-80 \mathrm{mV}$ and $-10 \mathrm{mV}$. Since the single-channel recordings were carried out with $312 \mathrm{msec}$ test depolarizations every $4 \mathrm{sec}$, most of the transitions would be expected to occur during the $3.7 \mathrm{sec}$ interval between pulses. The percentage of transitions expected during the depolarizations themselves can be calculated as follows. For a switching rate of $\sim 0.1 \mathrm{sec}^{-1}$, the likelihood of a transition amidst the depolarization would be $(0.3 \mathrm{sec})\left(0.1 \mathrm{sec}^{-1}\right)=0.03$ per pulse. This is comparable to the frequency of midsweep modal transitions we were able to detect. For example, some of the stray points in the $\left\langle t_{o}\right\rangle-\left\langle t_{c}\right\rangle$ plot in Figure $4 D$ can be accounted for by intrasweep transitions (see Fig. 4 caption for details). In general, the rate of occurrence of such transitions was low enough not to hamper seriously the analysis of gating kinetics on a sweep-by-sweep basis.

\section{Low- $\mathrm{p}_{0}$ openings can show larger unitary amplitude}

It is interesting to compare the voltage dependence of the unitary current within each mode. No difference is seen in the current amplitude of openings in the medium- and high- $p_{0}$, modes. However, the channel can show a larger unitary current amplitude in low- $p_{o}$ sweeps than in the medium- $p_{o}$ or high- $p_{o}$ sweeps. This can be seen in Figure 2 and is illustrated further by the records in Figure 11. The broken lines represent an estimate of the open levels in a group of medium- $p_{o}$ sweeps (Fig. $11 \mathrm{~A}$ ) and in a group of low- $p_{o}$ sweeps (Fig. $11 B$ ), all at $-10 \mathrm{mV}$ test potential. There is a consistent difference in unitary current amplitude: when openings in low- $p_{o}$ sweeps are clearly resolved, they attain values $\sim 0.2 \mathrm{pA}$ greater than unitary openings in the medium- $p_{o}$ sweeps. The difference is reproducible, and opposite of that expected 
A

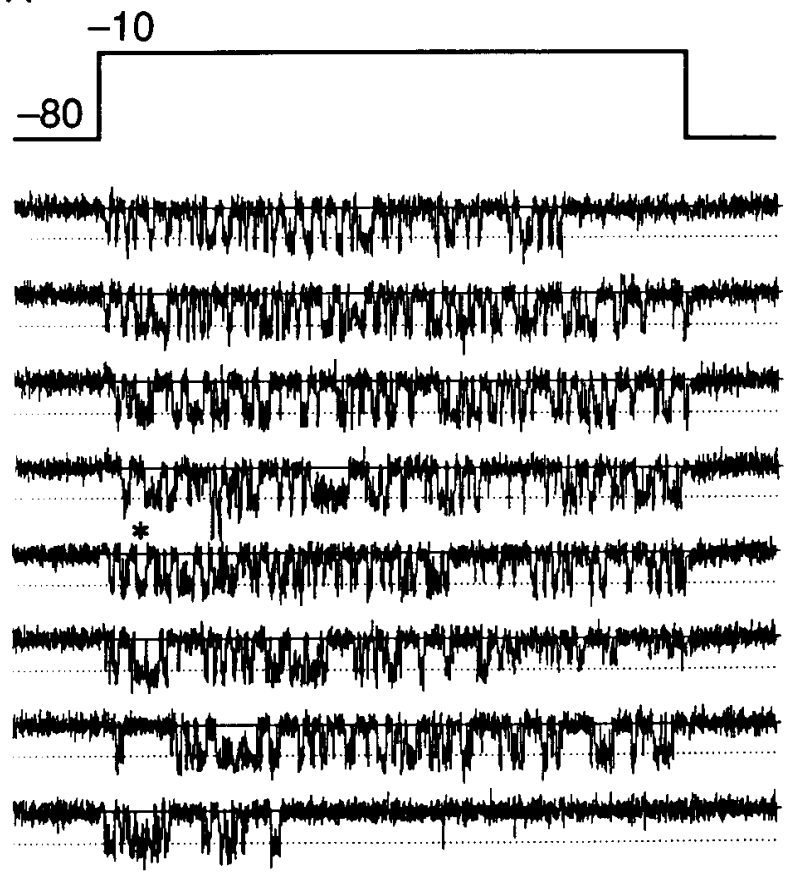

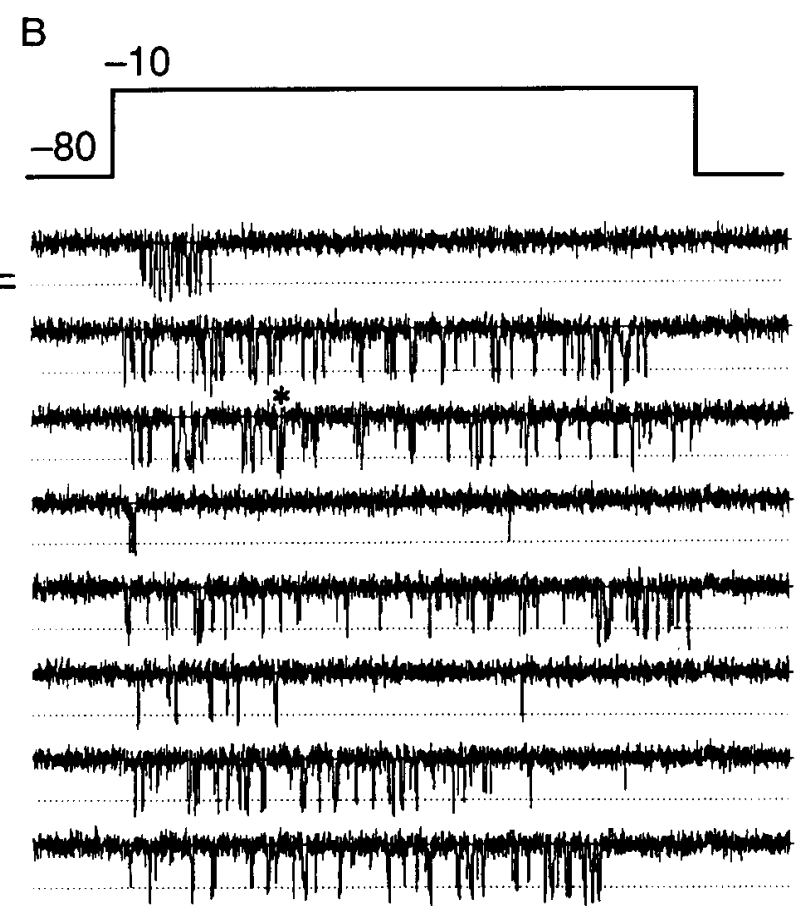

$2 \mathrm{pA} L$

$50 \mathrm{~ms}$
C

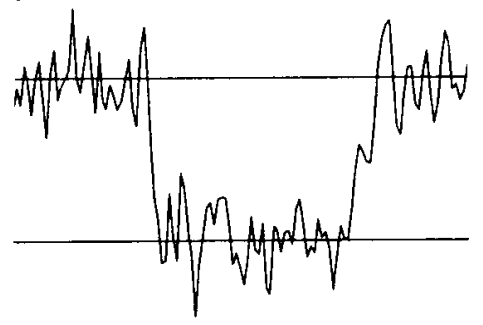

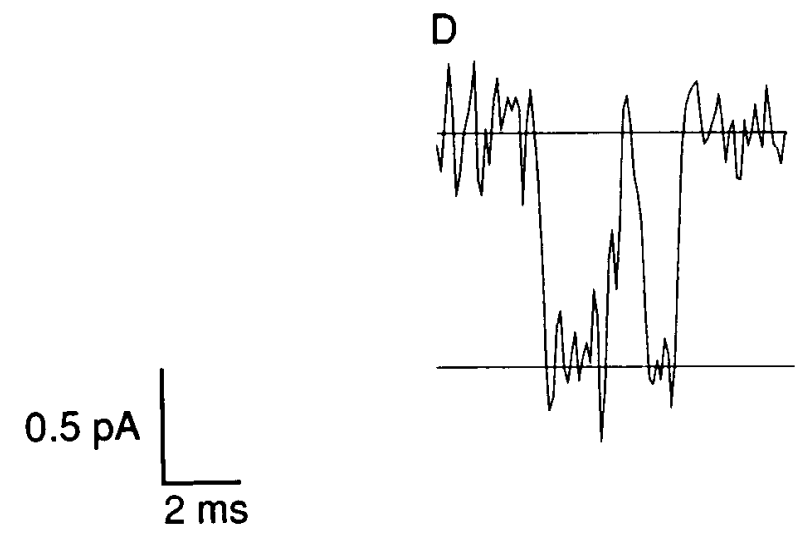

Figure 11. The single-channel conductance of the low- $p_{i}$ mode can differ from that of the other modes: selected leak-subtracted sweeps illustrating medium- $p_{0}$ gating $(A, C)$ and the low- $p_{o}$ gating $(B, D) . C$ and $D$ are selected channel openings taken from records in $A$ and $B$ as indicated by the asterisks, displayed on an expanded time scale to highlight the difference in current amplitude.

for imperfect resolution of brief openings due to bandwidth limitations. Indeed, when records are expanded along the time axis (Fig. $11 C, D$ ), it is evident that many of the low- $p_{o}$ openings are quite well resolved.

As illustrated in Figure $12 \mathrm{~A}$, the difference in unitary current is also apparent in all-points histograms of the current signal from the sweeps in Figure 11. The open channel current levels correspond to peaks of Gaussian distributions (arrows) of -0.72 $\mathrm{pA}$ for the medium $-p_{o}$ mode and $-0.95 \mathrm{pA}$ for the low $-p_{o}$ mode. Differences between the unitary current amplitude of the low$p_{o}$ mode and medium/high- $p_{o}$ modes were found consistently over a range of membrane potentials. Figure $12 \mathrm{~B}$ shows col- lected results from six patches. The unitary current amplitude for sweeps showing low- $p_{o}$ gating is at least $0.2 \mathrm{pA}$ larger than the corresponding values for high- and medium- $p_{\text {, }}$ sweeps at several test potentials. The single-channel conductance was found to be $\sim 18 \mathrm{pS}$ for both modes. While the $i(V)$ relations run parallel over a considerable range of potentials studied, it seems possible that the $i(V)$ curves would converge at strongly positive potentials.

The low- $p_{o}$ openings are themselves considerably smaller in unitary current amplitude than openings of L-type channels $(\sim 1.2-1.3 \mathrm{pA}$ at $-10 \mathrm{mV})$. L-type channels may also be distinguished by their resistance to inactivation by steady depo- 


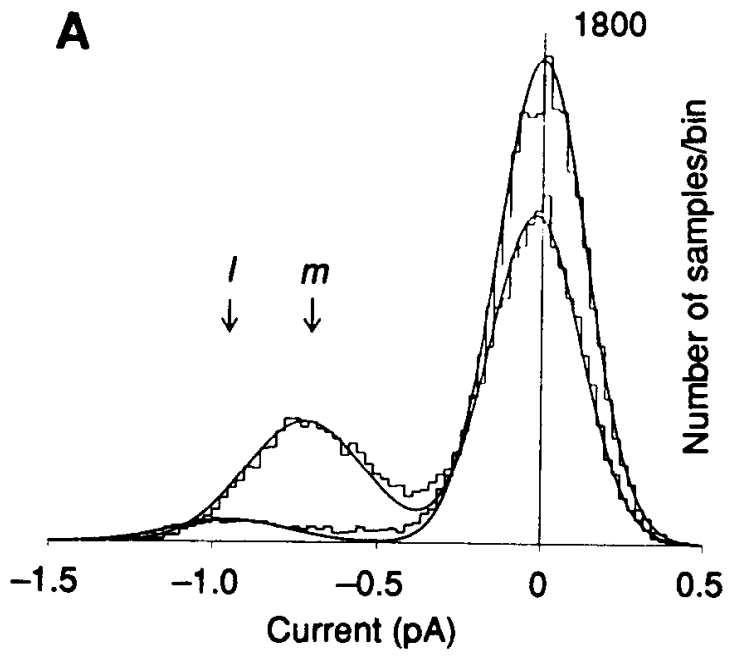

B

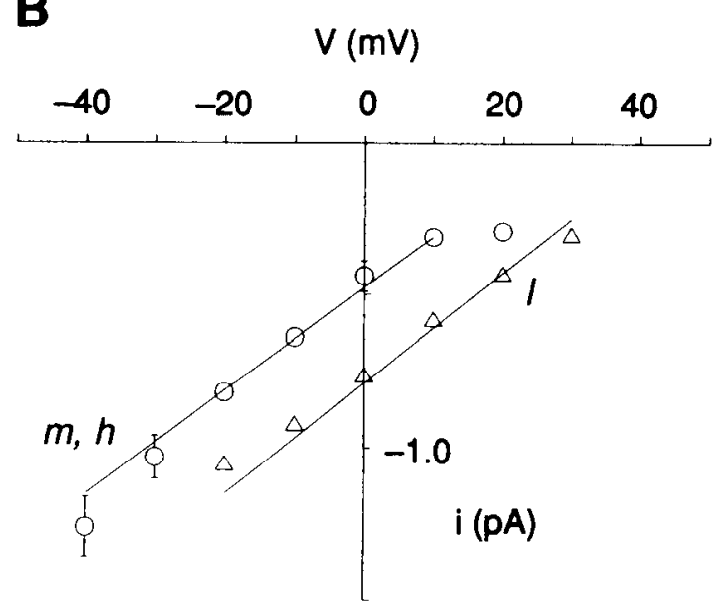

Figure 12. A, Amplitude histograms of the sweeps in Figure 11. The closed and open current levels were fitted individually by single Gaussian distributions; the sum of the fitted Gaussians is displayed. The peaks of the current amplitudes are marked by the arrows $\left(l\right.$, low $p_{o} ; m$, medium $p.) . B$, Unitary current-voltage relationships for low- $p_{o}$ behavior $(\triangle)$ and medium/high- $p$, behavior $(O)$. The current amplitude was averaged for a number of sweeps in each of five experiments, but the lines were drawn from the regression analysis performed with the individual experimental values. Single-channel current amplitudes of medium- and high- $p$, sweeps were grouped together since they showed no apparent difference. Both regression lines have slopes of $18 \mathrm{pS}$. The y-intercepts are $-0.49 \mathrm{pA}$ for the medium/high- $p_{0}$ modes and $-0.80 \mathrm{pA}$ for the low- $p_{o}$ mode.
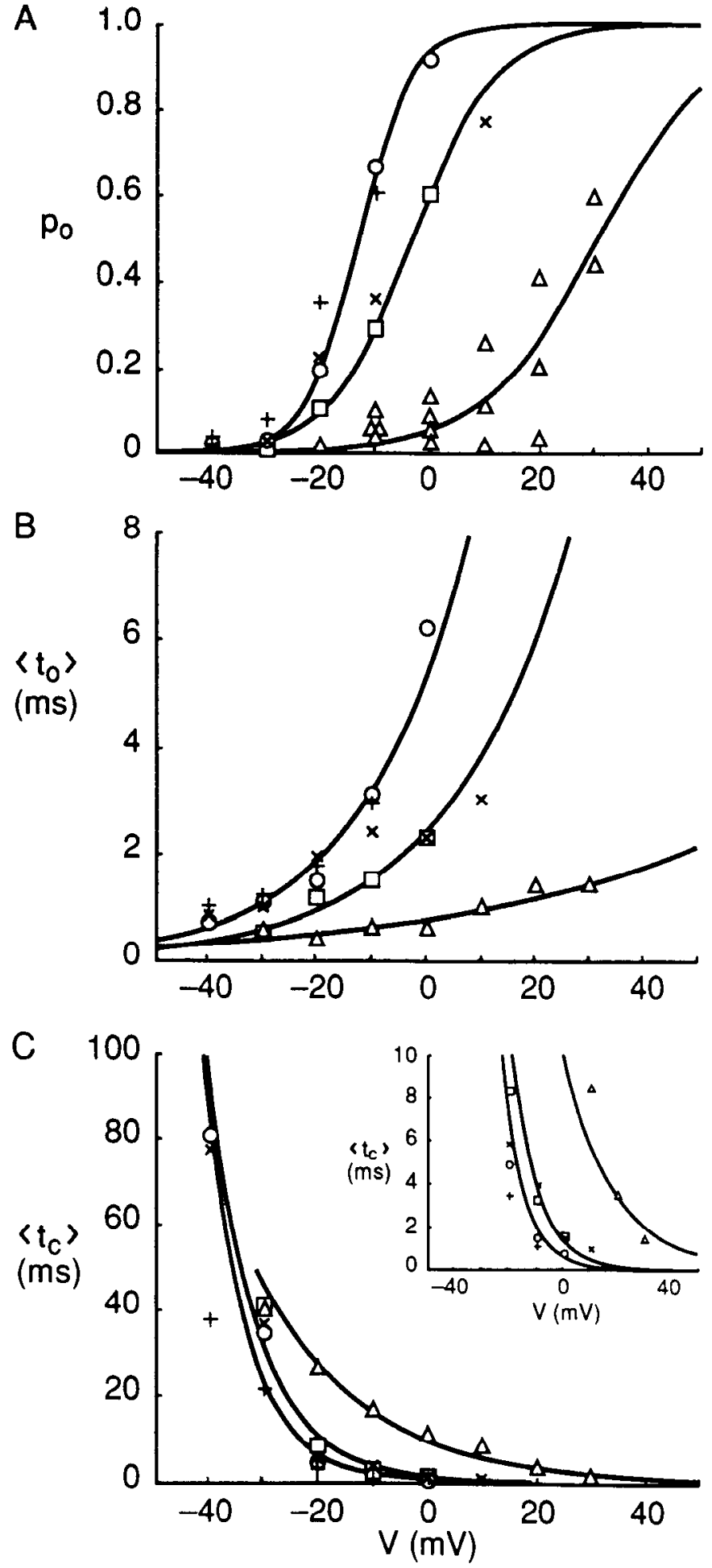

$(+$ and $x)$ in which medium- and high- $p_{0}$ were not clearly separated Although the curve fitting was performed without regard for these points, it is clear that they fall in the range of the curves for medium and high $p_{o} . B$, Voltage dependence of the mean open times. Symbols are as in $A$, except that the data for the low- $p_{0}$ mode have been averaged. The solid lines represent the exponential fits of the data donc by linear regression of data plotted on a logarithmic scale. An $e$-fold change in $\left\langle t_{o}\right\rangle$ is obtained for 50,22 , and $19 \mathrm{mV}$ for the low-, medium-, and high$p_{\text {o }}$ modes, respectively. $C$, Voltage dependence of the mean closed times. Symbols are as in $B$. The solid lines represent the exponential fits (linear regression of a logarithmic plot). An $e$-fold change in $\left\langle t_{c}\right\rangle$ is obtained for $19,9.3$, and $8 \mathrm{mV}$ for the low-, medium-, and high- $p_{0}$ modes, respectively. The inset shows $\left\langle t_{c}\right\rangle$ plotted on a vertically expanded scale. $7.8 \mathrm{mV})$ and the high- $p$ mode $\left(0 ; V^{\prime}=-13.2 \mathrm{mV} ; k=5 \mathrm{mV}\right)$. Also plotted for the sake of completeness are data from two other experiments 
larizations. We routinely applied steady depolarizations to -40 $\mathrm{mV}$ to verify that channel activity inactivated as expected for $\mathrm{N}$-type $\mathrm{Ca}^{2+}$ channels (but not L-type channels).

\section{Differences in voltage-dependent activation}

Figure 13 compares the voltage dependence of activation of the three modes. Figure $13 \mathrm{~A}$ shows individual $p_{0}$, values for low- $p_{o}$ activity from seven experiments (triangles). Three of these patches exhibited low- $p_{0}$ activity exclusively as judged by intermittent trials at $-10 \mathrm{mV}$. In the other patches, low- $p_{0}$ gating was isolated by a combination of characteristics, including unitary amplitude and gating kinetics, which make it appear quite different from the other modes over a wide range of potentials. Average values for $\left\langle t_{v}\right\rangle$ and $\left\langle t_{c}\right\rangle$ in the low- $p_{0}$ mode were derived from the same set of experiments and are plotted in Figure 13,B and $C$.

It was harder to separate medium- and high- $p_{0}$ activity at potentials beyond $-10 \mathrm{mV}$ because the $p_{o}$ increases so strongly in both modes. The analysis of $p_{o}(V)$ for these modes (Fig. 13A) relied heavily on two individual experiments containing long runs with exclusively medium- $p_{o}$ gating (squares) or high- $p_{o}$ gating (circles). The plot also includes data from two other experiments (represented by + and $x$ ) where it was more difficult to make a clear-cut distinction between medium- and high- $p_{0}$,gating at voltages other than $-10 \mathrm{mV}$. These data are included because they provide some information about the behavior of non-low- $p$, gating in aggregate.

Data for $p_{i}(V)$ were fit with a function derived from the Boltzmann distribution (Fig. 13A). The $p_{o}(V)$ curves differed in their midpoints $\left(V_{1 / 2}=-13.2,-3.2\right.$, and $\left.31 \mathrm{mV}\right)$ and Boltzmann slope factors $(k=5,7.8$, and $11 \mathrm{mV})$ for high-, medium-, and low- $p_{n}$ modes, respectively. Thus, there was a systematic progression in the position and steepness of the $p_{o}(V)$ relationships. The voltage dependence of the low- $p$, mode shows a relatively large separation from the other two modes, which fall fairly close to each other along the voltage axis.

The voltage dependence of the mean open times and mean closed times for each mode were fit with simple exponential functions (Fig. 13B,C). In the low- $p_{o}$ mode, $\left\langle t_{o}\right\rangle$ and $\left\langle t_{c}\right\rangle$ both displayed a shallower voltage dependence ( $e$-fold slope factors of $50 \mathrm{mV}$ and $19 \mathrm{mV}$, respectively) than in the other two modes (slope factors of $\sim 20 \mathrm{mV}$ and $\sim 8 \mathrm{mV}$ ).

\section{Discussion}

This study provides direct evidence for the existence of multiple modes of $\mathrm{N}$-type $\mathrm{Ca}^{2+}$ channel activation gating and a quantitative description of the open-closed kinetics within each mode. Our analysis at the level of unitary currents complements previous studies of whole-cell N-type currents that originally suggested the existence of "reluctant" and "willing" gating modes with different voltage dependence (Bean, 1989b; see also Elmslie et al., 1990; Ikeda, 1991; Kasai, 1992). We found that N-type channels exhibit at least three discrete patterns of gating, denoted as low-, medium-, and high- $p_{0}$, modes according to their probability of being in the opcn statc at $10 \mathrm{mV}$, a test potential where the different modes are particularly well resolved. The modes differ in the position and steepness of their voltage-dependent activation curves. Within each mode, the kinetics of gating were adequately described by a set of Markov states arranged in the same configuration $(\mathrm{C} 2-\mathrm{Cl}-\mathrm{O})$ as found in kinetic studies of other types of $\mathrm{Ca}^{2+}$ channels (e.g., Fenwick et al., 1982; Reuter et al., 1982; Brum et al., 1984). The rate constants connecting these states are markedly different from

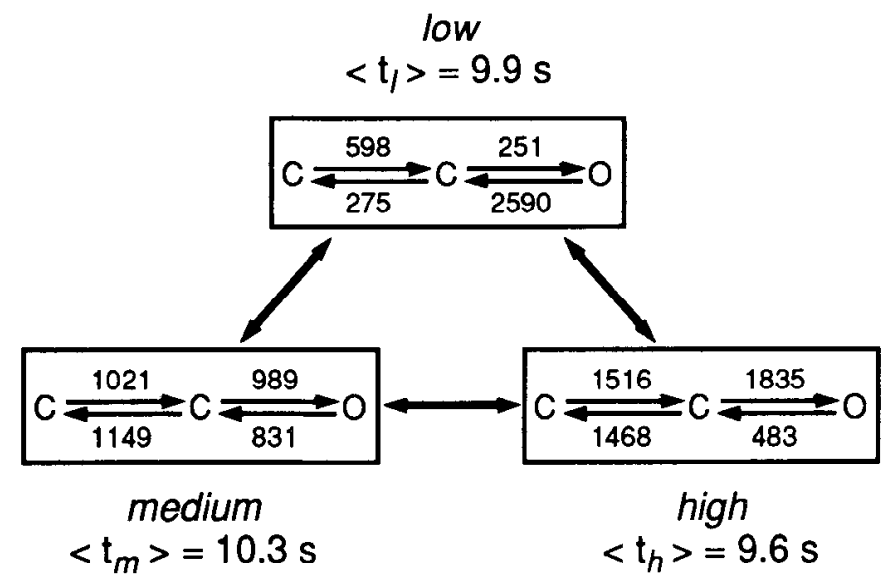

Figure 14. Summary of collected data on kinetic properties of the three modes. This scheme represents threc sets of C2-C1-O states, corresponding to each mode, in equilibrium with each other. The mean dwell times in each mode are indicated as $\left\langle t_{t}\right\rangle$, and so on, and were derived from the experimental data in Figure $6 A-C$. The rate constants (in sec ') for the transitions between closed and open states are arithmetic averages of individual values from 13 experiments. For each experiment, the rate constants were calculated from estimates of the average $p_{u}$ and of the areas and time constants of the exponential components of the open and closed time distributions (Fenwick et al., 1982).

one group of $\mathrm{C} 2-\mathrm{Cl}-\mathrm{O}$ states to the next (Fig. 14), thereby giving each mode its own distinctive kinetic signature.

Runs analysis demonstrated that $\mathrm{N}$-type $\mathrm{Ca}^{2+}$ channels undergo spontaneous but infrequent transitions between the various patterns of gating. The average dwell time in any individual mode is $\sim 10 \mathrm{sec}$, of the order of $10^{3}$ to $10^{4}$ times greater than mean durations of individual openings or closings within each mode. Thus, an N-type channel undergoes hundreds or thousands of open-closed transitions during a sojourn in a particular mode before spontaneously switching to a different gating pattern in another mode. Most transitions between modes occurred during the $3.7 \mathrm{sec}$ interval between depolarizations, although mid-sweep transitions were also observed (Fig. 10). All permutations of transitions have been observed, and there was no clear indication for preferred transitions among the active gating modes.

\section{Key discriminators between gating modes of $N$-type channels}

The combination of high-, medium-, and low- $p_{n}$, modes is more complex than the modal schemes proposed on the basis of wholecell recordings. Are three modes really necessary? This amounts

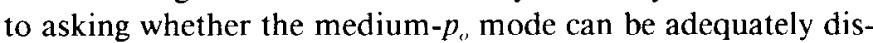
criminated from low $-p_{o}$ behavior on one hand and the high- $p_{\text {, }}$ mode on the other.

Medium- and high- $p_{o}$ modes were readily separated on the basis of their kinetic behavior. The $p_{o}$ values at $-10 \mathrm{mV}$ for medium- and high- $p_{o}$ modes are $\sim 0.3$ and $\sim 0.6$, respectively. In either case, there are typically scores of open and closed events during a $320 \mathrm{msec}$ depolarizing pulse. Thus, estimates of $\left\langle t_{0}\right\rangle$ and $\left\langle t_{c}\right\rangle$ for a given depolarization represent information about kinetic behavior that has already been signal averaged over the large number of events in that particular sweep. Each mean value carries with it an estimate of SEM (Fig. 3), so differences in mean values can be evaluated for statistical significance by conventional tests. In practice, medium- and high- $p_{o}$ modes differ significantly with respect to both $\left\langle t_{0}\right\rangle$ and $\left\langle t_{c}\right\rangle$.

The relative proportion of sweeps displaying high- $p_{n}$ gating is 
quite variable. In some patches, sweeps with high $p_{0}$ are in the majority (Fig. $4 C$ ), whereas in other cases, high- $p_{o}$ gating can be completely absent (Fig. $4 A$ ). The variability is convenient for the purposes of isolating high- $p_{\text {, }}$ gating as a distinct mode.

Medium- and low- $p_{0}$ gating were also separated by comparing mean open or closed dwell times, calculated for individual sweeps. Plots of $\left\langle t_{t}\right\rangle$ against $\left\langle t_{c}\right\rangle$ provided a rather clear separation of sweeps into two populations. The mean closed time was the most dramatic discriminator, being about fourfold longer for low- $p_{0}$, than for medium- $p_{0}$ gating.

The amplitude of single-channel currents was an additional discriminator. When well resolved, the unitary amplitude of low- $p_{o}$ openings was about $0.2 \mathrm{pA}$ larger than medium- or high$p_{o}$ openings (Figs. 11,12 ). It is important to note that the observed difference in conductance is contrary to that expected from bandwidth limitations, which would tend to make brief openings appear smaller.

The overall conclusion is that the medium- $p_{o}$ mode can be adequately distinguished from high- or low- $p_{o}$ behavior, supporting the use of a minimum of three active modes to describe $\mathrm{N}$-type channel gating.

\section{Is the modal scheme too simple?}

The possibility remains that further subdivision of the rapid gating modes will eventually become necessary. This seems unlikely for the high- $p_{0}$ mode because the open-closed characteristics are thoroughly documented and conform so closely to a conventional C2-C1-O scheme. Subdivision of low- $p_{o}$ seems much more likely. Indeed, there is a wide variability in $\left\langle t_{c}\right\rangle$ values among different single-channel patches that exceeds what might be expected from random sampling from a single homogeneous distribution (e.g., Fig. $4 B, D$ ).

Sweeps classified as medium- $p_{o}$ also show a wider spread on $\left\langle t_{b}\right\rangle-\left\langle t_{c}\right\rangle$ plots than expected from Monte Carlo simulations based on empirically derived C2-C1-O kinetics (Fig. 9). Some of the spread can be explained in terms of errors arising from sweepby-sweep analysis. When transitions between various modes occur partway through a sweep, it is likely that the swecp will have an intermediate value of $p_{o}$ and thereby be categorized (inappropriately) as medium $p_{o}$. In many cases, the misclassified sweep can be identified as an aberrant data point on the $\left\langle t_{0}\right\rangle-$ $\left\langle t_{c}\right\rangle$ plot, with verification by visual inspection of the trace (see Fig. 4 caption). However, the midsweep transitions are not always obvious, so no attempt was made to exclude hybrid sweeps.

An additional subclass of medium- $p_{\text {o }}$ gating may eventually be necessary to accommodate a pattern of activity characterized by brief openings ( $\sim 1 \mathrm{msec}$ ) and brief closings ( $\sim 1.5 \mathrm{msec})$. We referred to this as the "buzz mode," by analogy to a very rare pattern of activity of $\mathrm{Ca}^{2+}$-activated $\mathrm{K}^{+}$channels (McManus and Magleby, 1988). The $p_{o}$ is $\sim 0.4$, in the same range as the usual medium- $p_{o}$ gating, although the closed times are much shorter. This kind of behavior was found infrequently (in less than $10 \%$ of the patches), so no attempt was made to isolate it from more typical medium- $p_{o}$ behavior illustrated in Figure 4.

\section{Comparison with modal analysis of other types of channels}

Spontaneous changes in the pattern of gating have now been found for many kinds of channels. These include ligand-gated channels for glutamate (Patlak et al., 1979) and GABA (Macdonald and Twyman, 1991) as well as voltage-gated channels such as Na channcls (Patlak and Ortiz, 1986; Nilius, 1988; Moorman et al., 1990; Zhou et al., 1991) and $\mathrm{Ca}^{2+}$-activated
$\mathrm{K}^{+}$channels (Moczydlowski and Latorre, 1983; McManus and Magleby, 1988). Modal changes in both activation and inactivation kinetics have been reported. The inactivation gating modes found for Na channels (Patlak and Ortiz, 1986; Nilius, 1988; Moorman et al., 1990; Zhou et al., 1991) are somewhat similar to those found for $\mathrm{N}$-type $\mathrm{Ca}^{21}$ channels (Plummer and Hess, 1991; see Different Kinetic Patterns, above).

The gating behavior described in this article finds strongest analogies with modal gating of L-type channels, previously described in heart cells (Reuter et al., 1982; Hess et al., 1984; Mazzanti and DeFelice, 1990; Yue et al., 1990) and neurons (Nowycky et al., 1985b). For L-type channels, as in the present case, modes were used as a way of describing well-defined patterns of rapid gating that remained stationary over a large number of duty cycles of opening and closing. L-type channcls display three active modes, termed mode $0_{a}$, mode 1 , and mode 2 (Hess et al., 1984; Yue et al., 1990). In a qualitative way, these are analogous to low-, medium-, and high- $p_{o}$ modes of N-type channels. Note however, that L-type mode 2 shows much longer openings $(\tau \sim 20 \mathrm{msec}$ near $0 \mathrm{mV})$ and much slower deactivation $(\tau \sim 10 \mathrm{msec}$ near $-40 \mathrm{mV})$ than $\mathrm{N}$-type high- $p_{o}$. The most striking contrast is in the frequency of occurrence of the modal behavior under basal conditions. L-type mode 2 is relatively rarely expressed (in perhaps $0.1-1 \%$ of depolarizations; Hess et al., 1984; Mazzanti and DeFelice, 1990), while high- $p$, gating of $\mathrm{N}$-type channels can appear in a majority of sweeps (Fig. $4 \mathrm{C}$ ). The likelihood of an L-type channel visiting mode 2 is greatly increased by $\beta$-adrenergic stimulation acting through cAMPdependent protein kinase. The prevailing hypothesis is that channel phosphorylation favors mode 2 , just as depolarization promotes transitions between closed states and an open state (Yue et al., 1990). Put in more general terms, a mode may be viewed as an expression of a modulatory state of the channel, superimposed on the set of conformational states occupied during normal gating.

Modal switching can be regulated by membrane potential as well as hormones or neurotransmitters. Strong depolarizations promote transitions of L-type channels from mode 1 to mode 2 (Hoshi and Smith, 1987; Lee, 1987; Pietrobon and Hess, 1990; Artalejo et al., 1991). Facilitation of N-type currents has also been found in whole-cell recordings (EImslie et al., 1990; Ikeda, 1991). More experiments are needed to see if strong depolarizations favor switching among rapid gating modes of single $\mathrm{N}$-type channels.

\section{Impact of modal weighting on divalent cation entry}

Alterations in the proportion of time spent in the individual modes will strongly influence the functional contribution of $\mathrm{N}$-type channels to divalent cation entry. The total current can be expressed as the sum of currents generated by the individual modes, whose contribution varies with its modal weighting coefficient $\left(A_{\text {low }}\right.$, etc.):

$$
\begin{aligned}
& I_{\text {tolat }}=I_{\text {low }}+I_{\text {med }}+I_{\text {high }} \\
& I_{\text {total }}=A_{\text {low }} \cdot p_{o, \text { low }} \cdot i_{\text {low }}+A_{\text {med }} \cdot p_{o, \text { med }} \cdot i_{\text {med }}+A_{\text {high }} \cdot p_{o, \text { high }} \cdot i_{\text {high }}
\end{aligned}
$$

Consider the case of a depolarization to a fixed potential, for example, $-10 \mathrm{mV}$. The mean current in a high- $p_{o}$ sweep is $p_{o, \text { high }}$. $i_{\text {high }}=(0.65)(0.6 \mathrm{pA})=0.4 \mathrm{pA}$, while the current in a low- $p_{0}$ sweep is $(0.05)(0.8)=0.04 \mathrm{pA}$, an order of magnitude less. Thus, shifts from high- $p_{o}$ to low- $p_{o}$ gating would greatly reduce the ovcrall $\mathrm{Ca}^{2+}$ entry during a voltage-clamp pulse or a high-K ${ }^{1}$ depolarization. 
Our experiments show a large degree of spontaneous variability from patch to patch in the modal weighting coefficients ( $A_{\text {low }}$, etc.). This variability is interesting in light of previously reported differences in voltage-dependent activation curves derived from whole-cell tail current recordings (Bean, 1989b; Ikeda, 1991). Tail current activation curves based on our analysis of unitary N-type channel activity (data not shown) can be compared with published data from whole-cell recordings. A reconstructed activation curve based on the modal weights observed in a patch where high- $p_{\text {o }}$ behavior is dominant (Fig. 4D) closely resembles the control curve in frog sensory neurons (Bean, 1989b) or the behavior of rat sympathetic neurons dialyzed with GDP$\beta$-S (Ikeda, 1991). Conversely, the experiment of Figure $4 B$, which displays a majority of low- $p_{o}$ behavior, yields a simulated activation curve similar to data for rat sympathetic neurons dialyzed with $0.5 \mathrm{~mm} \mathrm{GTP- \gamma -S}$ (Ikeda, 1991). Evidently, activation of $\mathrm{G}$-proteins favors low- $p_{n}$ (reluctant) modal gating. One possible implication for our experiments is that the state of G-proteins or some other modulatory factor may vary widely from patch to patch or cell to cell even without the overt interventions used by Ikeda (1991).

Variability in the contribution of different modes has additional implications for identification of various $\mathrm{Ca}^{2+}$ current components in whole-cell recordings. Separation of N-type and L-type current components on the basis of voltage dependence of activation may be difficult if modal weighting is undetermined.

\section{Molecular and functional implications}

Taken together, these results support the idea that G-proteins influence the equilibrium between gating modes (Bean, 1989b; Elmslie et al., 1990; Ikeda, 1991; Kasai, 1992). How might this occur? (1) Our results demonstrate that the activity of individual channels is not controlled in an all-or-nothing manner, but can display multiple levels of $p_{v}$. This suggests that the G-protein does not merely block the channel. (2) The finding of at least three active modes of gating is also relevant to questions of mechanism. It docs not support the simple hypothesis whereby binding of a single G-protein molecule is tightly linked to a transition from willing to reluctant gating. Other possibilities remain open. For example, one-to-one binding of a $\mathrm{G}$-protein to an N-type channel might tilt the equilibrium between modes in favor of medium- and low- $p_{o}$ behavior. Alternatively, more than one G-protein may bind to a channel, and modal changes may be determined by the number of G-proteins bound (see Kurachi et al., 1990; Karschin et al., 1991). (3) Gating kinetics within each of the modes can be adequately described by a common kinetic scheme $(\mathrm{C} 2-\mathrm{Cl}-\mathrm{O})$. This suggests that gating in any mode arises from a common set of molecular conformations. (4) Switching between modes is relatively infrequent under the conditions of our experiments. This suggests that additional conformational changes in the channel, caused or favored by binding of $\mathrm{G}$-protein(s), occur on a slow time scale to alter the energetics of the $\mathrm{C} 2, \mathrm{C} 1$, or $\mathrm{O}$ states. Under basal conditions, in the absence of neurotransmitter, the level of $\mathrm{G}$-protein activation may be low enough to allow relatively long sojourns in high- or medium- $p$, mode. (5) The $p(V)$ relations for the various modes are not related to each other by a simple shift along the voltage axis, but also exhibit differences in steepness (Fig. 13). This implies that the modulatory action is not just a simple electrostatic effect, as in the casc of phosphorylation of $\mathrm{K}^{+}$channels (Perozo and Bezanilla, 1990). (6) The low- $p_{o}$ mode often displays a significantly larger single-channel conductance than the other modes, suggesting the modulatory mechanism can somehow alter the aspects of the channel molecule that control ion permeation as well as opening and closing.

Whatever the details of the mechanism, the possibility of G-protein-driven changes in mode offers wide latitude for neurotransmitter-dependent modulation. Alterations in the weighting of modes has been hypothesized as a mechanism of neurotransmitter-driven downmodulation (Bean, 1989b). We have direct evidence for this in the case of modulation by norepinephrine (Delcour et al., 1992).

Shifts between gating modes are a more subtle kind of modulation than simply blocking or unblocking the channel. The $\sim 40 \mathrm{mV}$ shift in voltage dependence between high- $p_{0}$, and low$p_{o}$ gating strongly reduces $p_{o}$ at $\sim 0 \mathrm{mV}$. However, it is important to recognize that even in low- $p_{o}$, open probability reaches a value of 0.5 at $+30 \mathrm{mV}$, near the peak of an action potential. Thus, the impact of the modulation will depend greatly on the pattern of electrical activity (Elmslie et al., 1990; Kasai, 1992; cf. Penington et al., 1991). Relief of inhibition could take place by accentuation of depolarization, favoring channel opening within the low- $p_{o}$ mode (Kasai, 1991), as well as by transitions from low- $p_{o}$, to medium- or high- $p_{o}$ modes (Bean, 1989b).

\section{References}

Aosaki T, Kasai H (1987) Characterization of two kinds of highvoltage-activated Ca-channel currents in chick sensory neurons: differential sensitivity to dihydropyridines and $\omega$-conotoxin GVIA. Pfluegers Arch 414:150-156.

Artalejo CR, Dahmer MK, Perlman RL, Fox AP (1991) Two types of $\mathrm{Ca}^{2+}$ currents are found in bovine chromaffin cells: facilitation is due to the recruitment of one type. J Physiol (Lond) 432:681-707.

Bean BP (1989a) Classes of calcium channels in vertebrate cells. Annu Rev Physiol 51:367-384.

Bean BP $(1989 \mathrm{~b})$ Neurotransmitter inhibition of neuronal calcium currents by changes in channel voltage dependence. Nature 340:153156.

Beech DJ, Bernheim L, Hille B (1992) Pertussis toxin and voltage dependence distinguish multiple pathways modulating calcium channels of rat sympathetic neurons. Neuron 8:97-106.

Brum G, Osterrieder W, Trautwein W (1984) $\beta$-Adrenergic increase in the calcium conductance of cardiac myocytes studied with the patch clamp. Pfluegers Arch 401:111-118.

Carbone E, Swandulla D (1989) Neuronal calcium channels: kinetics, blockade and modulation. Prog Biophys Mol Biol 54:31-58.

Colquhoun D, Sigworth FJ (1983) Fitting and statistical analysis of single-channel records. In: Single-channel recordings (Neher E, Sakmann B, eds), pp 191-263. New York: Plenum.

Delcour AH, Lipscombe D, Tsien RW (1992) Shifts in gating modes are involved in the noradrenergic down-modulation of $\mathrm{N}$-type $\mathrm{Ca}^{++}$ channels. Biophys J 61:A420.

Elmslic KS, Zhou W, Jones SW (1990) LHRH and GTP- $\gamma$-S modify calcium current activation in bullfrog sympathetic neurons. Neuron 5:75-80.

Fenwick EM, Marty A, Neher E (1982) Sodium and calcium channels in bovine chromaffin cells. J Physiol (Lond) 331:599-635.

Fox AP, Nowycky MC, Tsien RW (1987) Kinetic and pharmacological properties distinguishing three types of calcium currents in chick sensory neurones. J Physiol (Lond) 394:149-172.

Hamill OP, Marty A, Neher E, Sakmann B, Sigworth FJ (1981) Improved patch-clamp techniques for high resolution current recording from cells and cell-free membrane patches. Pfluegers Arch 391:85100.

Hess P (1990) Calcium channels in vertebrate cells. Annu Rev Neurosci 13:337-356.

Hess P, Lansman JB, Tsien RW (1984) Different modes of Ca channel gating behaviour favoured by dihydropyridine $\mathrm{Ca}$ agonists and antagonists. Nature 311:538-544.

Hirning LD, Fox AP, McCleskey EW, Olivera BM, Thayer SA, Miller RJ, Tsien RW (1988) Dominant role of $\mathrm{N}$-type $\mathrm{Ca}^{2+}$ channels in 
evoked release of norepinephrine from sympathetic neurons. Science 239:57-61.

Hoshi T, Smith SJ (1987) Large depolarization induces long openings of voltage-dependent calcium channels in adrenal chromaffin cells. $J$ Neurosci 7:571-580.

Ikeda SR (1991) Double-pulse calcium channel current facilitation in adult rat sympathetic neurones. J Physiol (Lond) 439:181-214.

Jackson MB, Wong BS, Morris CE, Lecar H, Christian CN (1983) Successive openings of the same acetylcholine receptor channel are correlated in open time. Biophys J 42:109-114.

Jones SW, Marks TN (1989a) Calcium currents in bullfrog sympathetic neurons. I. Activation kinetics and pharmacology. J Gen Physiol 94:151-167.

Jones SW, Marks TN (1989b) Calcium currents in bullfrog sympathetic neurons. II. Inactivation. J Gen Physiol 94:169-182.

Karschin A, Ho BY, Labarca C, Elroy-Stein O, Moss B, Davidson N, Lester HA (1991) Heterologously expressed serotonin 1A receptors couple to muscarinic $\mathrm{K}^{+}$channels in heart. Proc Natl Acad Sci USA 88:5694-5698.

Kasai $H$ (1991) Tonic inhibition and rebound facilitation of a neuronal calcium channel by a GTP-binding protein. Proc Natl Acad Sci US $\Lambda$ $88: 8855-8859$.

Kasai H (1992) Voltage- and time-dependent inhibition of neuronal calcium channels by a GTP-binding protein. J Physiol (Lond) 448: 189-200.

Kongsamut S, Lipscombe D, Tsien RW (1989) The N-type Ca channel in frog sympathetic neurons and its role in $\alpha$-adrenergic modulation of transmitter release. Ann NY Acad Sci 560:312-333.

Kowalik J, Osborn MR (1968) Methods for unconstrained optimization. New York: Elsevier.

Kurachi Y, Ito H, Sugimoto T (1990) Positive cooperativity in activation of the cardiac muscarinic $\mathrm{K}^{+}$channel by intracellular GTP. Pfluegers Arch 416:216-218.

Lee KS (1987) Potentiation of the calcium-channel currents of internally perfused mammalian heart cells by repetitive depolarization. Proc Natl Acad Sci USA 84:3941-3945.

Lipscombe D, Madison DV, Poenie M, Reuter H, Tsien RY, Tsien RW (1988) Spatial distribution of calcium channels and cy tosolic calcium transients in growth cones and cell bodies of sympathetic neurons. Proc Natl Acad Sci USA 85:2398-2402.

Lipscombe D, Kongsamut S, Tsien RW (1989) $\alpha$-Adrenergic inhibition of sympathetic neurotransmitter release mediated by modulation of N-type calcium channel gating. Nature 340:639-642.

Mazzanti M, DeFelice LJ (1990) Ca channel gating during cardiac action potentials. Biophys J 58:1059-1065.

Macdonald RL, Twyman RE (1991) Biophysical properties and regulation of $\mathrm{GABA}_{\mathrm{A}}$ receptor channels. The Neurosciences 3:219-235.

McManus OB, Magleby KL (1988) Kinetic states and modes of single large-conductance calcium-activated potassium channels in cultured rat skeletal muscle. J Physiol (Lond) 402:79-120.

McManus OB, Magleby KL (1989) Kinetic time constants independent of previous single-channel activity suggest Markov gating for a large conductance Ca-activated K channel. J Gen Physiol 94:10371070.

Miller RJ (1992) Voltage-sensitive $\mathrm{Ca}^{2}+$ channels. J Biol Chem 267: 1403-1406.

Moczydlowski E, Latorre R (1983) Gating kinetics of $\mathrm{Ca}^{2+}$-activated
$\mathrm{K}^{+}$channels from rat muscle incorporated into planar lipid bilayers Evidence for two voltage-dependent $\mathrm{Ca}^{2+}$ binding reactions. J Gen Physiol 82:511-542.

Moorman JR, Kirsch GE, Van Dongen AMJ, Joho RH, Brown AM (1990) Fast and slow gating of sodium channels encoded by a single mRNA. Neuron 4:243-252.

Nilius B (1988) Modal gating bchavior of cardiac sodium channels in cell-free membrane patches. Biophys J 53:857-862.

Nowycky MC, Fox AP, Tsien RW (1985a) Three types of neuronal calcium channel with different calcium agonist sensitivity. Nature 316:440-443.

Nowycky MC, Fox AP, Tsien RW (1985b) Long-opening mode of gating of neuronal calcium channels and its promotion by the dihydropyridine calcium agonist Bay K 8644. Proc Natl Acad Sci USA 82:2178-2182.

Patlak JB, Ortiz M (1986) Two modes of gating during late $\mathrm{Na}^{+}$ channel currents in frog sartorius muscle. J Gen Physiol 87:305-326.

Patlak JB, Gration KA, Usherwood PNR (1979) Single glutamate activated channels in locust muscle. Nature 478:643-645.

Penington NJ, Kelly JS, Fox AP (1991) A study of the mechanism of $\mathrm{Ca}^{2+}$ current inhibition produccd by serotonin in rat dorsal raphe neurons. J Neurosci 11:3594-3609.

Perozo E, Bezanilla F (1990) Phosphorylation affects voltage gating of the delayed rectifier $\mathrm{K}^{+}$channel by electrostatic interactions. Neuron 5:685-690.

Pietrobon D, Hess P (1990) Novel mechanism of voltage-dependent gating in L-type calcium channels. Nature 346:651-655.

Plummer MR, Hess P (1991) Reversible uncoupling of inactivation in N-type calcium channels. Nature 351:657-659.

Plummer MR, Logothetis DE, Hess P (1989) Elementary properties and pharmacological sensitivities of calcium channels in mammalian peripheral neurons. Neuron 2:1453-1463.

Regan LJ, Sah DWY, Bean BP (1991) $\mathrm{Ca}^{2+}$ channels in rat central and peripheral neurons: high-threshold current resistant to dihydropyridine blockers and $\omega$-conotoxin. Ncuron 6:269-280.

Reuter H, Stevens CF, Tsien RW, Yellen G (1982) Properties of single calcium channels in cultured cardiac cells. Nature 297:501-504.

Rittenhouse AR, Plummer MR, Hess P (1991) Microscopic heterogeneity of N-type calcium channel gating. Soc Neurosci Abstr 17:901.

Shuba YM, Teslenko VI, Savchenko AN, Pogorelaya NH (1991) The effect of permeant ions on single calcium channel activation in mouse neuroblastoma cells: ion-channel interaction. J Physiol (Lond) 443: $25-44$.

Stanley EF (1991) Single calcium channels on a cholinergic presynaptic nerve terminal. Neuron 7:585-591.

Tsien RW, Lipscombe D, Madison DV, Bley KR, Fox AP (1988) Multiple types of neuronal calcium channels and their selective modulation. Trends Neurosci 11:431-438.

Tsien RW, Ellinor PT, Horne WA (1991) Molecular diversity of voltage-dependent $\mathrm{Ca}^{2+}$ channels. Trends Pharmacol Sci 12:349-354.

Yue DT, Herzig S, Marban E (1990) $\beta$-Adrenergic stimulation of calcium channels occurs by potentiation of high-activity gating modes. Proc Natl Acad Sci USA 87:753-757.

Zhou J, Potts JF, Trimmer JS, Agnew WS, Sigworth FJ (1991) Multiple gating modes and the effect of modulating factors on the $\mu \mathrm{I}$ sodium channel. Neuron 7:775-785. 